\title{
Divector boson production in association with a Higgs boson at hadron colliders
}

\author{
Pankaj Agrawal, ${ }^{1,2,3, *}$ Debashis Saha $\odot,{ }^{1,2, \dagger}$ and Ambresh Shivaji ${ }^{4, \$}$ \\ ${ }^{1}$ Institute of Physics, Sainik School Post, Bhubaneswar 751005, India \\ ${ }^{2}$ Homi Bhabha National Institute, Training School Complex, Anushakti Nagar, Mumbai 400085, India \\ ${ }^{3}$ Department of Physics, Indian Institute of Technology Delhi, Hauz, Khas, New Delhi-110016, India \\ ${ }^{4}$ Indian Institute of Science Education and Research Mohali, \\ Knowledge City, Sector 81, S. A. S. Nagar, Manauli PO 140306, Punjab, India
}

(Received 2 February 2021; accepted 20 May 2021; published 24 June 2021)

\begin{abstract}
We consider the production of a Higgs boson in association with two electroweak vector bosons at hadron colliders. In particular, we examine $\gamma \gamma H, \gamma Z H, Z Z H$, and $W^{+} W^{-} H$ production at the LHC (14 TeV), HE-LHC (27 TeV), and FCC-hh (100 TeV) colliders. Our main focus is to estimate the gluongluon $(g g)$ channel $\left(g g \rightarrow V V^{\prime} H\right)$ contributions to $p p \rightarrow V V^{\prime} H\left(V, V^{\prime}=\gamma, Z, W\right)$ and compare them with corresponding contributions arising from the quark-quark $(q q)$ channel $\left(q \bar{q} \rightarrow V V^{\prime} H\right)$. Technically, the leading order $g g$ channel contribution to the $p p \rightarrow V V^{\prime} H$ cross section is a next-to-next-to-leading order correction in the strong coupling parameter, $\alpha_{s}$. In the processes under consideration, we find that in the $g g$ channel, $W^{+} W^{-} H$ has the largest cross section. However, the relative contribution of the $g g$ channel is more important for the $p p \rightarrow Z Z H$ production. At the FCC-hh, the $g g \rightarrow Z Z H$ contribution is comparable with the next-to-leading order QCD correction to $q q \rightarrow Z Z H$. We also compute the cross sections when $W$ and $Z$ bosons are polarized. In the production of $W^{+} W^{-} H$ and $Z Z H$, we find that the $g g$ channel contributes more significantly when the vector bosons are longitudinally polarized. By examining such events, one can increase the fraction of the $g g$ channel contribution to these processes. Further, we have studied beyond-thestandard-model effects in these processes using the $\kappa$-framework parameters $\kappa_{t}, \kappa_{V}$, and $\kappa_{\lambda}$. We find that the $g g$ channel processes $Z Z H$ and $W W H$ have a very mild dependence on $\kappa_{\lambda}$, but strong dependence on $\kappa_{t}$ and $\kappa_{V}$. The $q q$ channel processes mainly depend on $\kappa_{V}$. Dependence of the $g g$ channel contribution on $\kappa_{V}$ is stronger than that of the $q q$ channel contribution. Therefore, focusing on events with longitudinally polarized $W$ and $Z$ bosons, one can find stronger dependence on $\kappa_{V}$ that can help us measure this parameter.
\end{abstract}

DOI: 10.1103/PhysRevD.103.116020

\section{INTRODUCTION}

After the discovery of a Higgs-like resonance, with a mass of $125 \mathrm{GeV}$, at the Large Hadron Collider (LHC) in 2012, various properties of this new particle have been studied. The spin and parity measurements have established it as a $0^{+}$state at $99.9 \% \mathrm{CL}$ against alternative scenarios [1]. Couplings of this new particle with the fermions and gauge bosons predicted in the standard model (SM) are getting constrained as more and more data are being analyzed by the LHC experiments [2-4]. To this end,

\footnotetext{
*agrawal@iopb.res.in

†ebasaha@iopb.res.in

*ashivaji@iisermohali.ac.in
}

Published by the American Physical Society under the terms of the Creative Commons Attribution 4.0 International license. Further distribution of this work must maintain attribution to the author(s) and the published article's title, journal citation, and DOI. Funded by SCOAP ${ }^{3}$. the vector-boson fusion production of the Higgs boson, associated production of $V H(V=Z, W)$, and Higgs boson's decay into vector bosons set limits on the $H V V$ couplings $[5,6]$. The gluon-gluon $(g g)$ channel production

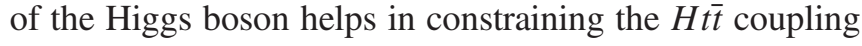
[6]. In addition, the evidence for the associated production of the Higgs boson with a top-quark pair $[7,8]$ will provide the direct measurement of $H t \bar{t}$ coupling. We still need to measure the trilinear and quartic Higgs self-couplings in order to know the form of the Higgs potential which will in turn reveal the exact symmetry breaking mechanism. The Higgs self-couplings can be probed directly in multi-Higgs production processes [9-11]. Recently, indirect methods of probing them at hadron and lepton colliders have also been proposed [12-14]. Similarly, the quartic couplings involving Higgs and vector bosons $H H V V$ are also not constrained independently. This coupling can be probed in the vector-boson fusion production of a Higgs boson pair $[15,16]$. In order to find the signals of new physics, it is important that we improve our theoretical predictions for 
the processes involving the Higgs boson at current and future colliders.

Loop-induced decay and scattering processes can play an important role in searching for new physics. In the presence of new physics (new particles and/or interactions), the rates for such processes can differ significantly from their standard model predictions. In this regard, many $g g$ channel scattering processes in the $2 \rightarrow 2$ and $2 \rightarrow 3$ categories have been studied [11,17-41]. In the present work, we are interested in loop-induced $g g$ channel contribution to $V V H \quad\left(\gamma \gamma H, \gamma Z H, Z Z H\right.$, and $\left.W^{+} W^{-} H\right)$ production. In QCD perturbation theory, the leading order $g g$ channel contribution to $p p \rightarrow V V H$ is a next-to-next-to-leading order (NNLO) contribution at the cross section level. Because of many electroweak couplings involved and the loop-induced nature of $g g \rightarrow V V H$ processes, their cross sections are expected to be small. However, they can be important at high energy hadron colliders like the $100 \mathrm{TeV}$ $p p$ collider such as the proposed hadronic Future Circular Collider (FCC-hh) facility at CERN [42] and the Super Proton-Proton Collider (SPPC) facility in China [43]. At such an energy scale, the gluon flux inside the proton becomes very large. In fact, for $\gamma \gamma H$, the $g g$ channel gives the dominant contribution.

Unlike the quark-quark contributions, which are mainly sensitive to $H V V$ couplings, the gluon-gluon contribution allows access to $H t \bar{t}, H H H$, and $H H V V$ couplings as well. Note that the processes under consideration are background to $p p \rightarrow H H$ when one of the Higgs bosons decays into $\gamma \gamma / \gamma Z / Z Z^{*}$ or $W W^{*}$ final states. The process $p p \rightarrow Z Z H$ is also a background to $p p \rightarrow H H H$ when two of the three Higgs bosons decay into $b \bar{b}$ final states. In this work, we present a detailed study of $g g \rightarrow \gamma \gamma H$ and $\gamma Z H$ for the first time in the SM. The $g g$ channel contribution to $Z Z H$ and $W W H$ in the SM have been studied in the past $[26,44,45]$. We have presented the $Z Z H$ and $W W H$ calculations in detail and have proposed methods to enhance the relative contribution of the gluon-gluon channel over the quarkquark channel. Since loop-induced processes are sensitive to new physics, we also study the effect of new physics in all $V V H$ processes using a common beyond the standard model (BSM) framework-the $\kappa$ framework. Going beyond the $\kappa$ framework, we have treated the $H H V V$ coupling independently and emphasized its effect in $\mathrm{ZZH}$ and $W W H$ processes. BSM study in a more sophisticated framework is desirable but it is beyond the scope of the present work.

Experimentally, $W$ - and Z-boson polarizations have been measured at hadronic colliders [46-48]. We also compute the cross sections for the processes when these bosons are polarized. For each process, the different production channels contribute predominantly to specific polarization configurations. This can help in enhancing the contribution of the $g g$ channel, as compared to the $q q$ channel. The $g g$ channel sometimes has a stronger dependence on the kappa parameters, in particular on $\kappa_{V}$. Therefore, an event sample with a larger $g g$ channel contribution can be helpful.

The paper is organized as follows. In Sec. II, we discuss the Feynman diagrams which contribute to $g g \rightarrow V V H$ amplitudes. The model independent framework to study new physics effects is outlined in Sec. III. In Sec. IV, we provide details on the calculation techniques and various checks that we have performed in order to ensure the correctness of our calculation. In Sec. V, we present numerical results in SM and BSM scenarios for all the $V V H$ processes. Finally, we summarize our results and conclude in Sec. VI.

\section{GLUON FUSION CONTRIBUTION TO $V V H$}

The $g g$ channel contribution to $p p \rightarrow V V H$ is due to a loop-induced scattering process mediated by a quark loop. The classes of diagrams contributing to $g g \rightarrow V V H$ processes are shown in Fig. 1. ${ }^{1}$ For convenience, the diagrams contributing to $g g \rightarrow W W H$ process are shown separately in Fig. 2. The $g g \rightarrow \gamma \gamma H$ process receives contributions only from the pentagon diagrams, while $\gamma Z H$ receives contributions from both the pentagon and box class of diagrams. In the case of $g g \rightarrow Z Z H, W W H$ processes, the triangle class of diagrams also contributes. We have taken all quarks but the top quark as massless. Therefore, the topquark contribution is relevant in diagrams where the Higgs boson is directly attached to the quark loop. In the diagrams where the Higgs boson does not directly couple to the quark loop, light quarks can also contribute. The complete set of diagrams for each process can be obtained by permuting external legs. These permutations imply that there are 24 diagrams in pentagon topology, six diagrams in each box topology, and two diagrams in each triangle topology. The diagrams in which only one type of quark flavor runs in the loop are not independent. Because of Furry's theorem, only half of them are independent [50]. This observation leads to a significant simplification in the overall calculation. This simplification, however, is not applicable to the $W W H$ case, where flavor changing interaction is involved in the quark loop. For example, see (a) and (b) in Fig. 2.

Thus, there are 12 independent pentagon diagrams [Fig. 1(a)] due to the top-quark loop contributing to the $g g \rightarrow \gamma \gamma H$ process. Similarly, the $g g \rightarrow \gamma Z H$ process receives contributions from 12 independent pentagon diagrams [Fig. 1(a)] due to the top-quark loop and three independent box diagrams [Fig. 1(b)] for each quark flavor. In principle, five light quarks $(u, d, c, s, b)$ and one heavy quark $(t)$ contribute. The box class of diagrams arise due to the $Z Z H$ coupling and has an effective box topology of the $g g \rightarrow \gamma Z^{*}$ amplitude. Furry's theorem, in this case, implies

\footnotetext{
${ }^{1}$ Feynman diagrams have been made using Jaxodraw [49].
} 


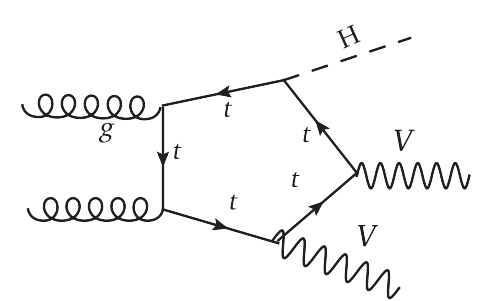

(a)

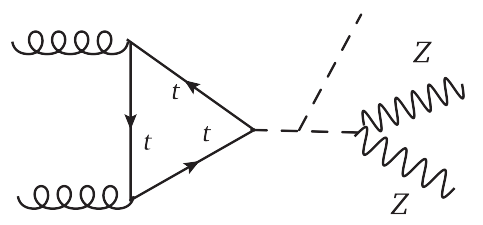

(d)



(b)

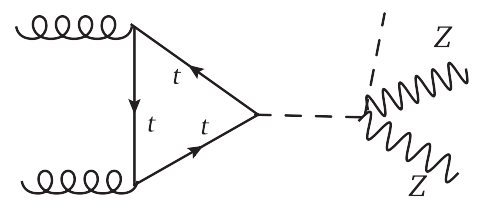

(e)



(c)

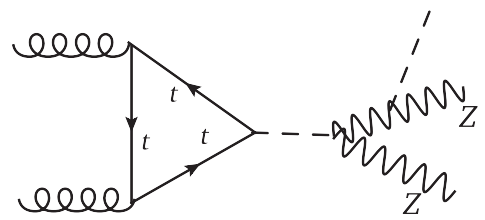

(f)

FIG. 1. Different classes of diagrams for $g g \rightarrow V V H, V=\gamma, Z$. In diagram (b), $q$ represents all quark flavors. Process $g g \rightarrow \gamma \gamma H$ receives contributions only from (a) type diagrams, while $g g \rightarrow \gamma Z H$ gets contributions from both (a) and (b) type diagrams. In the case of $Z Z H$, all the diagrams contribute; the diagrams (b) and (f) cover the situation in which $H$ is attached to a $Z$ boson.

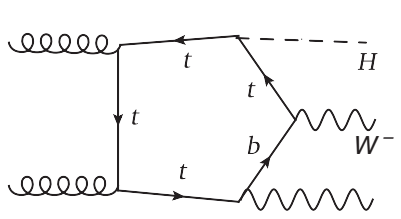

(a)

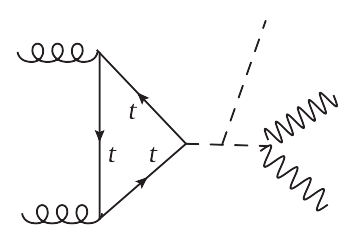

(e)

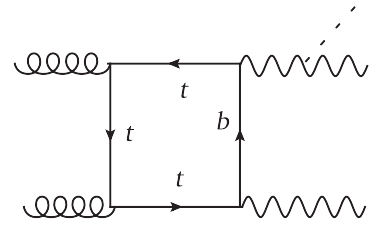

(b)

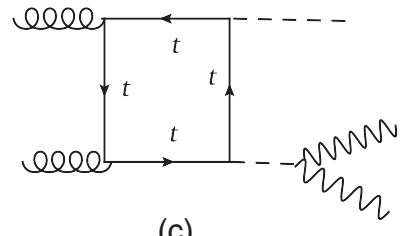

(c)



(d)

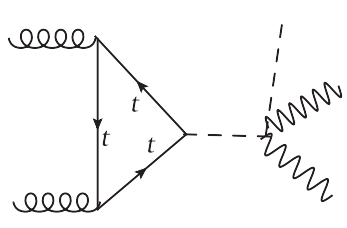

(f)

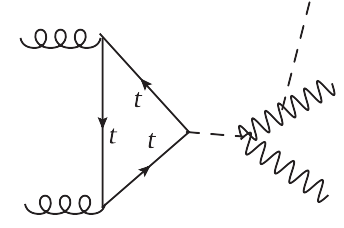

(g)

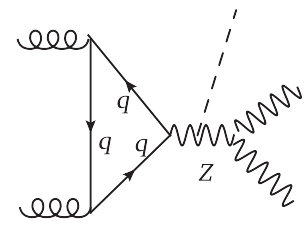

(h)

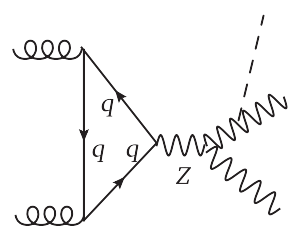

(i)

FIG. 2. Different classes of diagrams contributing to $g g \rightarrow W W H$ process. With respect to $Z Z H$, new classes of box and triangle diagrams appear due to $Z W W$ coupling. In (a) and (b), due to the flavor changing interaction of $W$ with quarks, both the quark flavors of a given generation enter in the loop. The diagrams (b), (g), and (i) cover the case when $H$ is attached to a $W$ boson.

that the axial-vector coupling of the $Z$ boson with quark does not contribute to the $g g \rightarrow \gamma Z H$ amplitude.

Like the $g g \rightarrow \gamma Z H$ process, the $g g \rightarrow Z Z H$ amplitude receives contributions from 12 independent pentagon diagrams with top quark in the loop [Fig. 1(a)]. However, there are six independent box diagrams with effective box topology of $g g \rightarrow Z Z^{*}$ amplitude for each quark flavor which covers the possibilities of the $H$ coupling with any of the two external $Z$ bosons [Fig. 1(b)]. Further, a new box type contribution arises which has effective box topology of $g g \rightarrow H H^{*}$ amplitude [Fig. 1(c)]. Once again there are three such independent diagrams with only the top quark in the loop. In addition to that, there are 4 independent triangle diagrams with a top quark in the loop and which have an effective triangle topology of $g g \rightarrow H^{*}$ amplitude [Figs. 1(d), 1(e), and 1(f)]. In $g g \rightarrow Z Z H$ amplitude, the Furry's theorem implies that the vector and axial-vector coupling of the $Z$ boson with quarks can contribute at quadratic level only.

Among all $V V H$ amplitudes, the structure of the $g g \rightarrow$ $W W H$ amplitude is the most complex. Because of the involvement of flavor changing interactions in Figs. 2(a) and 2(b), the Furry's theorem is not applicable to these diagrams. Therefore, 24 independent pentagon diagrams contribute to the $g g \rightarrow W W H$ process for each generation of quarks. However, since we neglect Higgs coupling with light quarks including the $b$ quark, there are only 12 nonzero independent pentagon diagrams. In Fig. 2(b), all 
the three quark generations contribute. Taking into account the possibility of Higgs boson coupling with any of the two external $W$ bosons, there are a total of 12 independent box diagrams of type (b) for each generation. In diagrams (a) and (b), the axial-vector coupling of $W$ with quarks contributes at quadratic as well as at linear level. Like in the $g g \rightarrow Z Z H$ process, there are three independent box diagrams of type (c). Because of $Z W W$ coupling, a new box contribution of type (d) having effective box topology of $g g \rightarrow H Z^{*}$ amplitude appears. Furry's theorem for diagram (d) implies that the vector coupling of $Z$ with quarks does not contribute to the amplitude. The same explains the absence of a similar box diagram due to $\gamma W W$ coupling. Further, there are four independent triangle diagrams with the top-quark loop [Figs. 2(e), 2(f), and 2(g)] as in the case of the $g g \rightarrow$ $\mathrm{ZZH}$ process. A new type of three independent triangle diagrams for each quark flavor with effective triangle topology of $g g \rightarrow Z^{*}$ amplitude appears, once again due to $Z W W$ coupling [Figs. 2(h) and 2(i)]. These triangle diagrams are anomalous and they can receive contribution only from the third generation quarks as the bottom and top quarks have very different masses. This is indeed the case for (h) type diagrams. However, we find that (i) type diagrams do not contribute. This is explained in the Appendix.

\section{BSM PARAMETRIZATION}

Measuring the couplings of the Higgs boson with fermions, gauge bosons, and with itself is an important aspect of finding the signatures of new physics at colliders. With the help of the data collected so far at the LHC, we now know couplings of the Higgs boson with the top quark with an accuracy of 10\%-20\% and with vector bosons with an accuracy of $10 \%$ at $1 \sigma$ [51]. The Higgs self-couplings, on the other hand, are practically unconstrained [52].

To study the new physics effects in $V V H$ processes, we take the simplest approach of modifying the SM-like couplings only, also known as the kappa framework for the parametrization of new physics $[53,54]$. In this framework, no new Lorentz structures and no new interaction vertices appear. The LHC experiments have interpreted the data using this framework so far. The couplings of our interest are $H t \bar{t}$, $H V V, H H H$, and $H H V V$. Out of these couplings, $g g \rightarrow \gamma \gamma H$ is sensitive to only $H t \bar{t}$ coupling. The $H V V$ coupling affects all other processes. The couplings $H H H$ and $H H V V$ affect only $g g \rightarrow V V H, V=Z, W$ processes.

The modification in these couplings due to new physics is implemented through scale factor $\kappa_{i}$ for various couplings of the Higgs boson in the SM. In the kappa framework, there are three such scale factors, namely, $\kappa_{t}$ for Higgs coupling with the top quark, $\kappa_{V}$ for Higgs coupling with vector bosons $\left(\kappa_{H Z Z}=\kappa_{H W W}=\kappa_{V}\right),{ }^{2}$ and $\kappa_{\lambda}$ for Higgs coupling with itself. Since in the SM both

\footnotetext{
${ }^{2}$ Note that in the SM, the tree level interaction vertices $H \gamma \gamma$ and $H \gamma Z$ do not exist.
}

$H V V$ and $H H V V$ couplings are related, the scaling of $H H V V$ coupling is also parametrized by $\kappa_{V}$. In a more generic BSM framework, the $H H V V$ coupling, in principle, can be independent of $H V V$ coupling.

In the presence of BSM effects, the amplitudes for the $g g$ channel processes depend on $\kappa_{t}, \kappa_{V}$, and $\kappa_{\lambda}$ as follows:

$$
\begin{aligned}
\mathcal{M}^{\mathrm{BSM}}(g g \rightarrow \gamma \gamma H)= & \kappa_{t} \mathcal{M}_{\mathrm{PEN}}^{\mathrm{SM}} \\
\mathcal{M}^{\mathrm{BSM}}(g g \rightarrow \gamma Z H)= & \kappa_{t} \mathcal{M}_{\mathrm{PEN}}^{\mathrm{SM}}+\kappa_{V} \mathcal{M}_{\mathrm{BX}_{1}}^{\mathrm{SM}} \\
\mathcal{M}^{\mathrm{BSM}}(g g \rightarrow Z Z H)= & \kappa_{t} \mathcal{M}_{\mathrm{PEN}}^{\mathrm{SM}}+\kappa_{V} \mathcal{M}_{\mathrm{BX}}^{\mathrm{SM}}+\kappa_{t}^{2} \kappa_{V} \mathcal{M}_{\mathrm{BX}}^{\mathrm{SM}} \\
& +\kappa_{t} \kappa_{V} \kappa_{\lambda} \mathcal{M}_{\mathrm{TR}}^{\mathrm{SM}}+\kappa_{t} \kappa_{V} \mathcal{M}_{\mathrm{TR}_{2}}^{\mathrm{SM}} \\
& +\kappa_{t} \kappa_{V}^{2} \mathcal{M}_{\mathrm{TR}}^{\mathrm{SM}} \\
\mathcal{M}^{\mathrm{BSM}}(g g \rightarrow W W H)= & \kappa_{t} \mathcal{M}_{\mathrm{PEN}}^{\mathrm{SM}}+\kappa_{V} \mathcal{M}_{\mathrm{BX}_{1}}^{\mathrm{SM}_{1}}+\kappa_{t}^{2} \kappa_{V} \mathcal{M}_{\mathrm{BX}}^{\mathrm{SM}_{2}} \\
& +\kappa_{t} \mathcal{M}_{\mathrm{BX}_{3}}^{\mathrm{SM}}+\kappa_{t} \kappa_{V} \kappa_{\lambda} \mathcal{M}_{\mathrm{TR}_{1}}^{\mathrm{SM}} \\
& +\kappa_{t} \kappa_{V} \mathcal{M}_{\mathrm{TR}_{2}}^{\mathrm{SM}}+\kappa_{t} \kappa_{V}^{2} \mathcal{M}_{\mathrm{TR}_{3}}^{\mathrm{SM}} \\
& +\kappa_{V} \mathcal{M}_{\mathrm{TR}_{4}}^{\mathrm{SM}_{2}}
\end{aligned}
$$

In the above, the amplitude $\mathcal{M}_{i}^{\mathrm{SM}}$ is related to one of the diagram classes displayed in Fig. 1 (Fig. 2 for $W W H$ ). This can be easily identified by looking at $\kappa$ factors in front of the amplitude. Note that in the $W W H$ amplitude, $\mathcal{M}_{\mathrm{TR}_{4}}^{\mathrm{SM}}$ includes both (h) and (i) type diagrams of Fig. 2. This parametrization does not affect the gauge invariance of the amplitudes with respect to the gluons as it will become clear in the next section. The standard model prediction can be obtained by setting $\kappa_{t}=\kappa_{V}=\kappa_{\lambda}=1$. Thus, except in $g g \rightarrow \gamma \gamma H$, we can expect nontrivial interference effects on total and differential cross sections for $g g \rightarrow V V H$ processes due to new physics in the $\kappa$ framework.

\section{CALCULATION AND CHECKS}

The calculation of quark-loop diagrams is carried out using a semiautomated in-house package OVReduce [55] which allows the calculation of any one-loop amplitude with a maximum of five propagators in the loop. The main steps involved in our calculation are as follows: quark-loop trace evaluation, one-loop tensor reduction to master integrals, and evaluation of master integrals. Trace calculation and simplification of the amplitude are done using symbolic manipulation software, FORM [56]. Tensor reduction of one-loop amplitudes into one-loop master integrals is done numerically following the method of OldenborghVermaseren [57]. Further, the one-loop master integrals are also calculated numerically using the ONELOOP package [58]. More details on this can be found in [23]. We perform the calculation in $4-2 \epsilon$ space-time dimensions to regulate ultraviolet (UV) and infrared (IR) singularities of one-loop master integrals. Since the couplings of $Z$ and $W$ bosons with quarks involve $\gamma_{5}$, the trace calculation needs special 
care. We have used four-dimensional properties of $\gamma_{5}$ in the calculation. This works because the SM is anomaly free. We have chosen unitary gauge for the calculation of the amplitudes.

The amplitude calculation for each process can be efficiently organized using prototype amplitudes for each class of diagrams. For example, amplitudes for all the 12 independent pentagon diagrams in the $g g \rightarrow \gamma \gamma H$ process can be obtained using only one prototype pentagon amplitude. Similarly, prototype amplitudes can be identified for each topology contributing to each process. The full amplitude for each process is a function of external momenta and polarization vectors/helicities. Because of huge expressions of the amplitudes, we calculate helicity amplitudes and the squaring of the amplitude for each process is done numerically. The number of helicity amplitudes for $g g \rightarrow \gamma \gamma H, \gamma Z H, Z Z H$, and $W W H$ processes are 16, 24, 36, and 36, respectively.

There are a number of checks that we have performed in order to ensure the correctness of the amplitudes. We have checked that the amplitudes are separately UV and IR finite. In $4-2 \epsilon$ dimensions, these divergences appear as poles in $1 / \epsilon$ (for UV and IR) and $1 / \epsilon^{2}$ (for IR only). Each pentagon diagram is UV finite. This we expect from the naive power counting. The individual box diagram is not UV finite; however, the full box amplitude, in each class, is UV finite. The UV finiteness of triangle amplitudes holds for each diagram. One-loop diagrams with all massive internal lines are IR finite, as expected. Thus, an IR finiteness check is relevant to the diagrams with massless quarks in the loop. This includes box class of diagrams of Fig. 1(b) in $g g \rightarrow$ $\gamma Z H$ and $Z Z H$. In the $g g \rightarrow W W H$ case, potentially IR divergent diagrams include Figs. 2(a), 2(b), 2(h), and 2(i). Unlike UV, the IR finiteness holds for each diagram [23].

We have also checked the gauge invariance of the amplitudes with respect to the external gluons. For that we numerically replace the gluon polarization vector $\epsilon^{\mu}(p)$ by its four momentum $p^{\mu}$ and expect a gauge invariant amplitude to vanish. We find that the gauge invariance check holds for each class of diagrams. This is expected because different box and triangle topologies for each process arise due to the existence of various electroweak couplings. This is a very strong check on our calculation which is organized using only prototype amplitudes. However, this check cannot verify relative signs between different classes of diagrams. In order to verify such relative signs, one needs to perform a gauge invariance check in electroweak theory, which is a nontrivial task. ${ }^{3}$ We rather rely on cross-checking the calculation using different methods and tools. We have compared our matrix element for each process with those calculated using MadLoop [60] and have found an excellent agreement. Being process

\footnotetext{
${ }^{3}$ A wrong relative sign between different class of diagrams may lead to violation of unitarity in certain processes [59].
}

specific, our code is efficient and provides greater flexibility when producing phenomenological results.

Numerical predictions for cross section and kinematic distributions are obtained using Monte Carlo techniques for phase space integration. We use AMCI [61] package for Monte Carlo phase space integration which is based on VEGAS [62] algorithm and allows parallelization of phase space point generation and matrix-element computation using PVM software [63].

\section{NUMERICAL RESULTS}

The cross section and kinematic distributions for $p p \rightarrow$ $V V H$ processes in SM and in BSM constitute the main results of this section. The numerical results are produced using following basic selection cuts unless stated otherwise,

$$
\begin{aligned}
p_{T}^{\gamma} & >50 \mathrm{GeV}, \quad\left|\eta^{\gamma}\right|<2.5, \\
\Delta R_{\gamma \gamma} & >0.4, \quad\left|y^{H, Z, W}\right|<5 .
\end{aligned}
$$

The results for the $g g$ channel processes are calculated using the CT14LO [64] parton distribution function and partonic center-of-mass energy $(\sqrt{\hat{s}})$ is chosen as a common scale for renormalization $\left(\mu_{R}\right)$ and factorization $\left(\mu_{F}\right)$. The results are obtained for three different choices of collider energies: $\sqrt{s}=14,27$, and $100 \mathrm{TeV}$. From the phenomenological point of view, we will focus on $p_{T}(H)$ and $M(V V)$ distributions.

We compare the $g g$ channel contribution to $p p \rightarrow V V H$ with the contribution arising from the $q q$ channels. The $q q$ channel contribution at LO and NLO (QCD) is calculated using MadGraph5_aMC@NLO [60] in a five flavor scheme for all but $W W H$ production. The $q q$ channel contribution to $W W H$ production is instead calculated in the four flavor scheme. ${ }^{4}$ The LO $q q$ channel contributions are pure electroweak processes and they do not depend on $\alpha_{s}$. For LO and NLO (QCD) results, we use CTEQ14LO and CT14NLO parton distribution functions, respectively [64]. The scale choice is same as in the $g g$ channel calculation. In both $g g$ and $q q$ channel calculations, the scale uncertainties are estimated by varying $\mu_{R}$ and $\mu_{F}$ independently by a factor of 2 . We quote only minimum and maximum uncertainties thus obtained.

To quantify the relative importance of the $g g$ channel contribution in processes dominated by the $q q$ channel, we define the following ratio:

$$
R_{1}=\frac{\sigma_{g g}^{\mathrm{VVH}, \mathrm{LO}}}{\sigma_{q q}^{\mathrm{VVH}, \mathrm{NLO}}-\sigma_{q q}^{\mathrm{VVH}, \mathrm{LO}}} .
$$

\footnotetext{
${ }^{4}$ For $W W H$ production, currently MadGraph5_amC@NLO cannot produce NLO correction to the $b b$ channel.
} 
This ratio compares the leading order $g g$ channel contribution with NLO-QCD corrections in the $q q$ channel. Recall that technically $g g$ channels contribute at NNLO. Similarly, at a differential level we define another ratio,

$$
R_{2}=\frac{\left.\frac{d \sigma}{d X}\right|_{g g} ^{\mathrm{VVH}, \mathrm{LO}}}{\left.\frac{d \sigma}{d X}\right|_{q q} ^{\mathrm{VVH}, \mathrm{NLO}},},
$$

where $X$ denotes a kinematic variable.

As mentioned in Sec. III, the BSM effects are parametrized in terms of scale factors $\kappa_{t}, \kappa_{V}$, and $\kappa_{\lambda}$. In order to compare their relative importance, we vary them independently by $10 \%$ about their SM values. Further, we comment on the effect of $\kappa_{\lambda}$ and $\kappa_{H H V V}$ (the scale factor for the $H H V V$ coupling $^{5}$ ) which are least constrained at present, in $\mathrm{ZZH}$ and $W W H$ processes.

\section{A. Predictions for the $p p \rightarrow \gamma \gamma H$ process}

The cross section for this process is dominated by the $g g$ channel. In the $q q$ channel, only bottom-quark initiated subprocess contribute to $\gamma \gamma H$ production. However, this cross section is quite small, owing to small bottom Yukawa coupling. In Table I, we compare the $g g$ and $q q$ channel contributions to the hadronic cross section at 14,27, and $100 \mathrm{TeV}$ colliders. The results are with minimum $50 \mathrm{GeV}$ transverse momentum of photons. We find that the $g g$ channel contribution increases 40 times as the collider center-of-mass energy goes from 14 to $100 \mathrm{TeV}$. Because of a small cross section, this process cannot be observed at the HL-LHC; FCC-pp will be more suitable. The $g g$ channel contribution becomes important at a higher center-of-mass energy collider; as in this case, smaller partonic momentum fractions $(x)$ are accessible, where gluon flux is significantly large. The scale uncertainties on the cross sections for the $g g$ channel are in the range of 20\%-30\%. It is clear from the table that the $q q$ channel contribution is negligible compared to the $g g$ channel contribution. It is merely $1 \%$ of the $g g$ channel contribution even after including the NLOQCD corrections.

In Fig. 3, we have plotted $p_{T}$ distributions for hardest photon, next-to-hardest photon, and Higgs in the left figure, and diphoton invariant mass distribution $[M(\gamma \gamma)]$ in the right figure for the $100 \mathrm{TeV}$ collider (FCC-hh). The $p_{T}$ distributions for them peak around 150,90 , and $70 \mathrm{GeV}$, respectively. We find that the tail of $p_{T}(H)$ is softer than that of photons. The $M(\gamma \gamma)$ distribution shows an interesting feature-it has two peaks. The right peak occurs at around $350 \mathrm{GeV}$, exhibiting the $t \bar{t}$ threshold effect in the distribution. To verify that the second peak is indeed due to $t \bar{t}$ threshold effect, we changed in our code the value of $m_{t}$

\footnotetext{
${ }^{5}$ Note this is different from $k_{V}$, which scales both $H V V$ and $H H V V$ couplings at the same time.
}

TABLE I. A comparison of different perturbative orders in QCD coupling contributing to $p p \rightarrow \gamma \gamma H$ hadronic cross section at $\sqrt{s}=14,27$, and $100 \mathrm{TeV}$.

\begin{tabular}{lrrr}
\hline \hline$\sqrt{\mathrm{s}}(\mathrm{TeV})$ & $\sigma_{g g}^{\gamma \gamma \mathrm{H}, \mathrm{LO}}[\mathrm{ab}]$ & $\sigma_{g g}^{\gamma \gamma \mathrm{H}, \mathrm{LO}}[\mathrm{ab}]$ & \multicolumn{1}{c}{$\sigma_{g g}^{\gamma \gamma \mathrm{H}, \mathrm{NLO}}[\mathrm{ab}]$} \\
\hline 14 & $5.36_{-20 \%}^{+28 \%}$ & $0.033_{-14 \%}^{+13 \%}$ & $0.046_{-6 \%}^{+5 \%}$ \\
27 & $22.0_{-19 \%}^{+22 \%}$ & $0.153_{-17 \%}^{+15 \%}$ & $0.234_{-7 \%}^{+5 \%}$ \\
100 & $220.1_{-21 \%}^{+27 \%}$ & $1.4_{-20 \%}^{+20 \%}$ & $2.25_{-8 \%}^{+5 \%}$ \\
\hline \hline
\end{tabular}

from 173 to $200 \mathrm{GeV}$ and the second peak was found to get shifted to $400 \mathrm{GeV}$.

As mentioned before, this process is a background to double Higgs production process when one of the Higgs bosons decays into a photon pair. To manage the background one usually looks at the " $\gamma \gamma b \bar{b}$ " final state, instead of " $b \bar{b} b \bar{b}$, , as the signature of the double Higgs boson production. At a $100 \mathrm{TeV}$ collider, while the cross section for the $g g \rightarrow \gamma \gamma H$ production, with the cuts in Eq. (5), is about $220 \mathrm{ab}$, the cross section for $g g \rightarrow H H \rightarrow \gamma \gamma H$, with the same set of cuts, is about $2600 \mathrm{ab}$. From the right panel of Fig. 3, it can be seen that the cross section for $\gamma \gamma H$ production in the bin from 120 to $140 \mathrm{GeV}$ is about $3 \mathrm{ab}$. On the other hand, all the cross sections for $g g \rightarrow H H \rightarrow$ $\gamma \gamma H$ are concentrated in a very narrow width around the mass of Higgs, $125 \mathrm{GeV}^{6}$ As a result, $g g \rightarrow \gamma \gamma H$ is an insignificant background to the process $g g \rightarrow H H \rightarrow \gamma \gamma H$.

Regarding anomalous coupling contributions, we note that as only pentagon diagrams contribute to the process $g g \rightarrow \gamma \gamma H$, its cross section scales as $\kappa_{t}^{2}$. So a $10 \%$ change in $\kappa_{t}$ will change the cross section and distributions by about $20 \%$. For the $q q$ channel process, the cross section is too small. It depends on $\kappa_{b}$, which we do not change from the standard model value.

\section{B. Predictions for the $p p \rightarrow \gamma Z H$ process}

Unlike the $\gamma \gamma H$ case, the $\gamma Z H$ production receives dominant contribution from the $q q$ channel. With $p_{T}^{\gamma}>50 \mathrm{GeV}$, the $g g$ channel contributions to $\gamma \mathrm{ZH}$ production at 14, 27, and $100 \mathrm{TeV}$ colliders are 4, 16, and 168 $\mathrm{ab}$, respectively. The corresponding values for the LO $q q$ channel contribution are 689, 1733, and $7498 \mathrm{ab}$, respectively. From Table II, it can be seen that $R_{1}$, which is the ratio of the $g g$ channel contribution to NLO correction in the $q q$ channel, is as small as 0.06 for the $100 \mathrm{TeV}$ collider, and even smaller for HE-LHC (27 TeV) and LHC (14 TeV). The scale uncertainties for the $g g$ channel are around $20 \%$ while those for the $q q$ channel at NLO are in the range of $2 \%-3 \%$. A larger scale dependence in the $g g$ channel

\footnotetext{
${ }^{6}$ In the right panel of Fig. 3, at $125 \mathrm{GeV}$, rather than showing a very narrow Breit-Wigner distribution, we have shown the total cross section for $g g \rightarrow H H \rightarrow \gamma \gamma H$ by a single vertical line.
} 

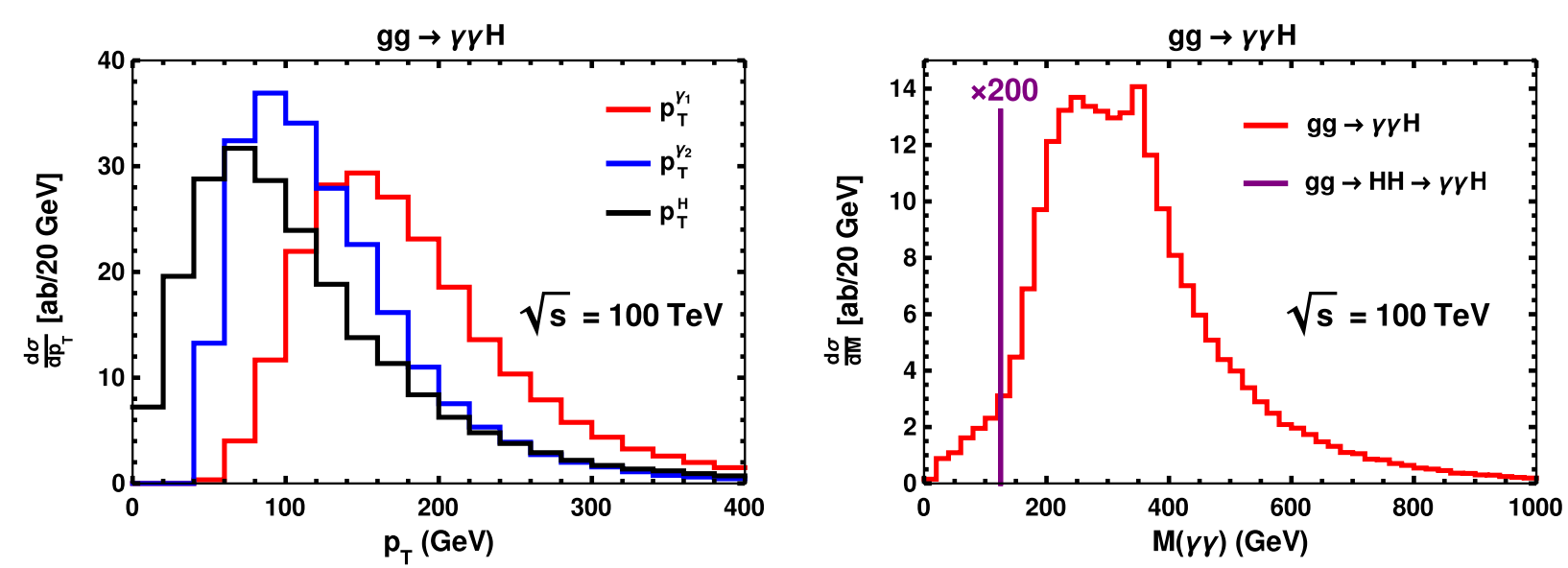

FIG. 3. Kinematic distributions for $g g \rightarrow \gamma \gamma H$ process in the SM at $100 \mathrm{TeV}$. In the $p_{T}$ distribution plot, $\gamma_{1}$ and $\gamma_{2}$ refer to the hardest and second hardest photons in $p_{T}$, respectively. In the right plot, we show $M(\gamma \gamma)$ distribution for $g g \rightarrow \gamma \gamma H$. In addition, the total cross section for the $g g \rightarrow H H \rightarrow \gamma \gamma H$ process has been shown at $125 \mathrm{GeV}$. " $\times 200$ " implies that the height of purple vertical line should be multiplied by a factor of 200 in order to get the correct cross section for the $g g \rightarrow H H \rightarrow \gamma \gamma H$ process.

contribution can be attributed to the presence of a higher power of the $\alpha_{s}$ factor in the $g g$ amplitudes.

In Table III, the effect of various $p_{T}^{\gamma}$ cuts in $g g$ and $q q$ channels has been shown. As the cut on $p_{T}^{\gamma}$ increases, the $q q$ channel cross section decreases faster than the $g g$ channel. In going from a 50 to $200 \mathrm{GeV}$ cut, the cross section of the $g g$ channel decreases roughly by a factor of 6 , while that of the $q q$ channel decreases by a factor of 9 . Thus, the relative contribution from the $g g$ channel can be enhanced with the help of a harder $p_{T}^{\gamma}$ cut. We find that the $p_{T}(H)$ cuts have the opposite effect i.e., the $g g$ channel is favored at low $p_{T}(H)$.

In Fig. 4, we have displayed $p_{T}$ distributions for the final state particles on the left, and $\gamma Z$-pair invariant mass distribution on the right for the $100 \mathrm{TeV}$ collider. The $p_{T}$ distributions peak around $100 \mathrm{GeV}$ while the $M(\gamma Z)$ distribution peaks around $200 \mathrm{GeV}$. Like the case of the $g g \rightarrow \gamma \gamma H$ process as a background to $g g \rightarrow H H \rightarrow \gamma \gamma H$, the $g g \rightarrow \gamma Z H$ process is also an insignificant background to $g g \rightarrow H H \rightarrow \gamma Z H$. This is because at a $100 \mathrm{TeV}$ collider, with the cuts in Eq. (5), the cross section for $g g \rightarrow H H \rightarrow$ $\gamma Z H$ is about $2000 \mathrm{ab}$, while the cross section for $g g \rightarrow$ $\gamma Z H$ process is about $170 \mathrm{ab}$. Moreover, all the cross sections for the $g g \rightarrow H H \rightarrow \gamma Z H$ process congregate around the mass of the decaying Higgs boson, $125 \mathrm{GeV}^{7}$, while, as can be seen from the right panel of the Fig. 4, the cross section for the $g g \rightarrow \gamma Z H$ process in the bin from 120 to $140 \mathrm{GeV}$ is about $3 \mathrm{ab}$. However, the $q q$ channel for the $\gamma \mathrm{ZH}$ production may act as an important background for the $g g \rightarrow H H \rightarrow \gamma Z H$ process.

In Fig. 5, we show $p_{T}(H)$ distributions for different classes of diagrams - pentagon, box, and sum of their

\footnotetext{
${ }^{7}$ However, instead of showing a very narrow Breit-Wigner distribution for the Higgs' decay, we have depicted the total cross section at $125 \mathrm{GeV}$ by a single vertical line.
}

individual contributions, their interference, and total at the $100 \mathrm{TeV}$ collider. The contribution of the box diagrams is more than the pentagon diagrams mainly because of the light quark contributions. The interference effect between the pentagon and box diagrams has kinematic dependence. We find that in the region of our kinematic interest, it is always destructive and, near the peak, its effect is close to $-30 \%$.

Since the $g g \gamma Z^{*}$-type box amplitude does not depend on the axial-vector coupling of the off-shell longitudinal $Z$ boson with the quarks, the top-quark contribution is not very significant at the level of the total cross section. This is shown in the right panel of Fig. 5. We can see that in the tail

TABLE II. A comparison of different perturbative orders in QCD coupling contributing to the $p p \rightarrow \gamma Z H$ hadronic cross section at $\sqrt{s}=14,27$, and $100 \mathrm{TeV}$. $R_{1}$ compares the $g g$ channel contribution with the correction at NLO and it is defined in Eq. (A6).

\begin{tabular}{lccrc}
\hline \hline$\sqrt{\mathrm{s}}(\mathrm{TeV})$ & $\sigma_{g g}^{\gamma \mathrm{ZH}, \mathrm{LO}}[\mathrm{ab}]$ & $\sigma_{g g}^{\gamma \mathrm{ZH}, \mathrm{LO}}[\mathrm{ab}]$ & $\sigma_{g g}^{\gamma \mathrm{ZH}, \mathrm{NLO}}[\mathrm{ab}]^{2}$ & $R_{1}$ \\
\hline 14 & $4.0_{-20 \%}^{+26 \%}$ & $689_{-0.2 \%}^{+0 \%}$ & $909_{-1.3 \%}^{+1.7 \%}$ & 0.02 \\
27 & $16_{-17 \%}^{+22 \%}$ & $1773_{-3.6 \%}^{+3.6 \%}$ & $2349_{-2.1 \%}^{+1.7 \%}$ & 0.03 \\
100 & $168_{-19 \%}^{+21 \%}$ & $7498_{-9.4 \%}^{+8.8 \%}$ & $10430_{-3.8 \%}^{+2.2 \%}$ & 0.06 \\
\hline \hline
\end{tabular}

TABLE III. Effect of $p_{T}^{\gamma}$ cut on the cross section of $p p \rightarrow \gamma Z H$ production at the $100 \mathrm{TeV}$ collider (FCC-hh).

\begin{tabular}{lccc}
\hline \hline$p_{T, \text { min }}^{\gamma}$ & $g g \rightarrow \gamma \mathrm{ZH}$ & $q q \rightarrow \gamma \mathrm{ZH}(\mathrm{LO})$ & $q q \rightarrow \gamma \mathrm{ZH}(\mathrm{NLO})$ \\
$(\mathrm{GeV})$ & {$[\mathrm{ab}]$} & {$[\mathrm{ab}]$} & 10430 \\
\hline 50 & 168 & 7498 & 4072 \\
100 & 95 & 2812 & 2069 \\
150 & 47 & 1366 & 1190 \\
200 & 28 & 765 & 0 \\
\hline \hline
\end{tabular}



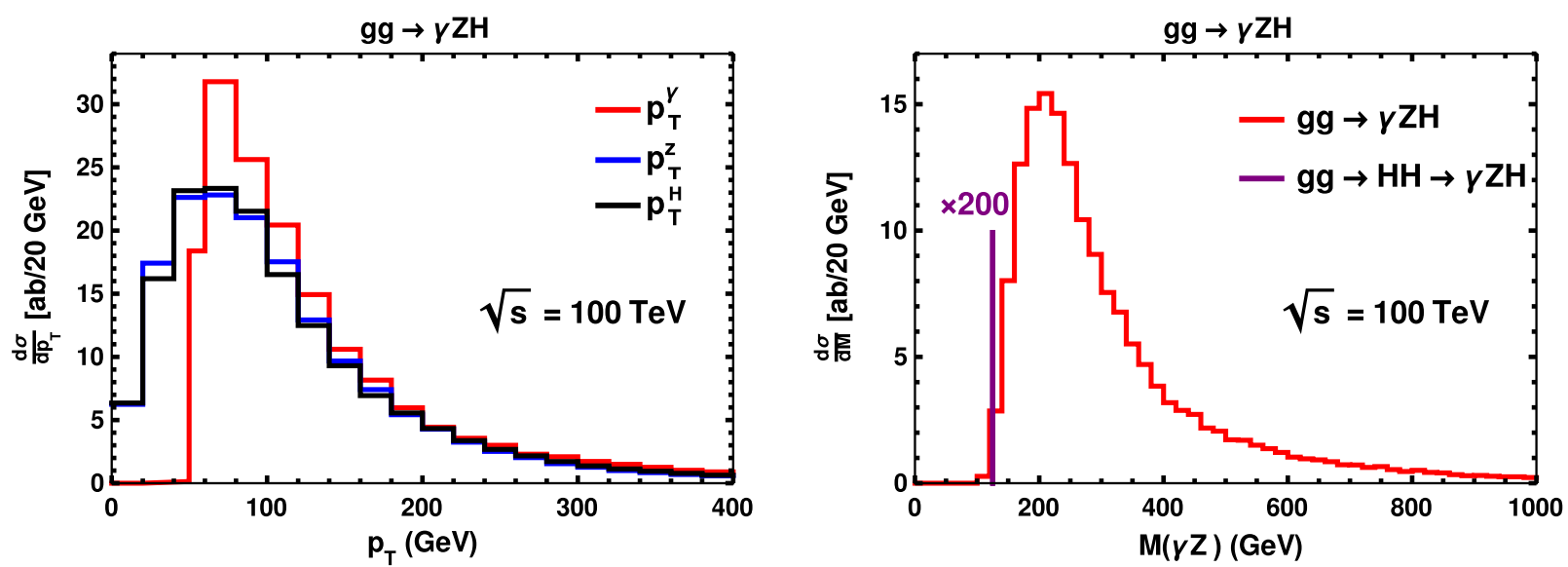

FIG. 4. Kinematic distributions for $g g \rightarrow \gamma Z H$ in the $\mathrm{SM}$ at $100 \mathrm{TeV}$. The purple vertical line in the right plot at $125 \mathrm{GeV}$ shows the total cross section for the process $g g \rightarrow H H \rightarrow \gamma Z H . \times 200$ means that the height of the purple vertical line needs to be scaled by a factor of 200 to get the correct cross section for the $g g \rightarrow H H \rightarrow \gamma Z H$ process.


FIG. 5. Left: The contribution of pentagon (blue) and box (green) diagrams, as well as their squared sum, interference, and total contribution to $p_{T}(H)$ distributions for the $g g \rightarrow \gamma Z H$ process at the $100 \mathrm{TeV}$ FCC-hh collider. Right: The effect of excluding the topquark contribution from the diagrams in Fig. 1(b) to the full amplitude.

where the top quark is effectively light, the cross section increases by about $20 \%$.

We have noted that the relative importance of the gluon fusion channel can be enhanced by applying higher $p_{T}(\gamma)$ cuts. To distinguish the $g g$ channel contribution from the dominant $q q$ channel, one can use the polarized cross sections and distributions. In Fig. 6, we have displayed the LO cross sections for various helicity states of the final state particles, $\gamma$ and $Z$ bosons. The figure also shows the contribution of various polarization states of the initial state particles. We cannot measure the polarization of the initial state particles that are in a bound state, the proton. However, experimentally, one can measure the $Z$ boson polarization [46-48]. The polarization of the photon has been measured by the LHCb Collaboration in $b$ baryon's decay[65-71]. At a $100 \mathrm{TeV}$ collider, the contribution of the $g g$ channel process to the production of $\gamma Z H$ is only $2.2 \%$. However, if we look at those final states where the photon and $Z$ boson have the same transverse polarization, then this ratio increases to $10 \%-$ $11 \%$. (The $q q$ channel makes the largest contribution when the $Z$ boson is longitudinally polarized.) This is a nontrivial contribution and can be measured if enough integrated luminosity is available. In Fig. 7, we have plotted the Higgs boson and Z-boson $p_{T}$ distributions. By making appropriate cuts on the small and large $p_{T}$ of these particles, we can further enhance the $g g$ channel contribution.

Turning to the effect of anomalous couplings, we find that the $g g$ channel shows very small dependence on the $\kappa_{t}$, 

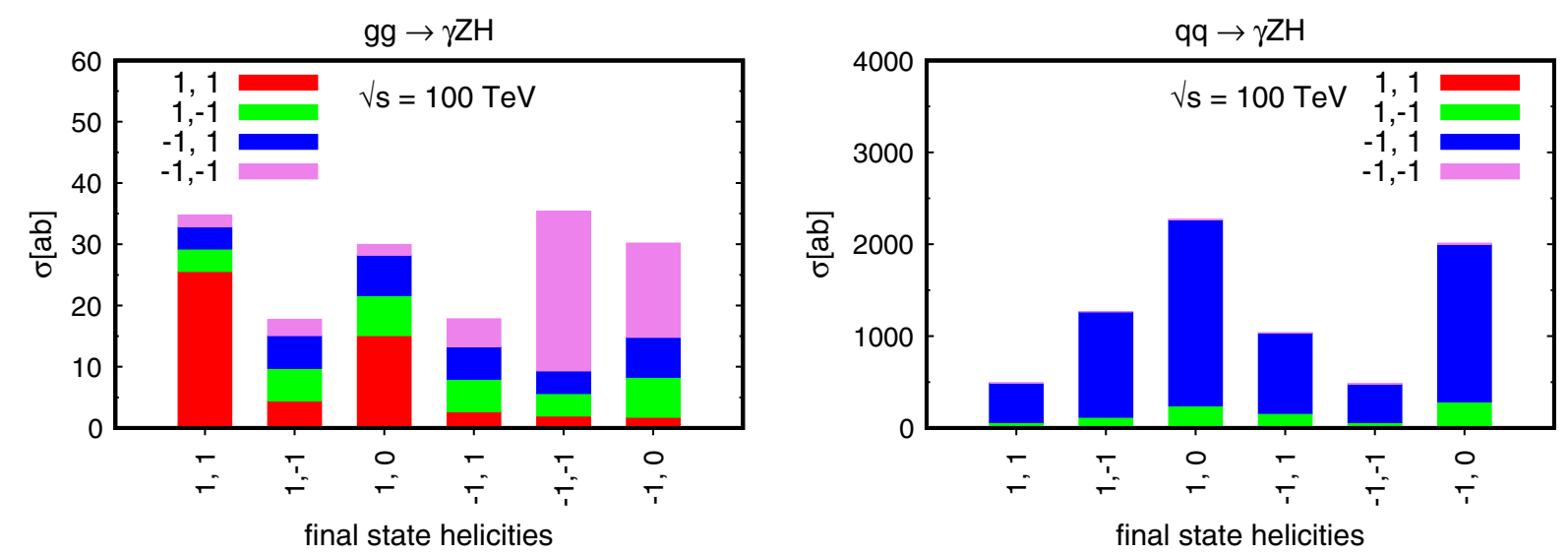

FIG. 6. LO cross section for $\gamma Z H$ production in different helicity configurations in the $g g$ (left) and $q q$ (right) channels. Legends correspond to different helicities of initial states.
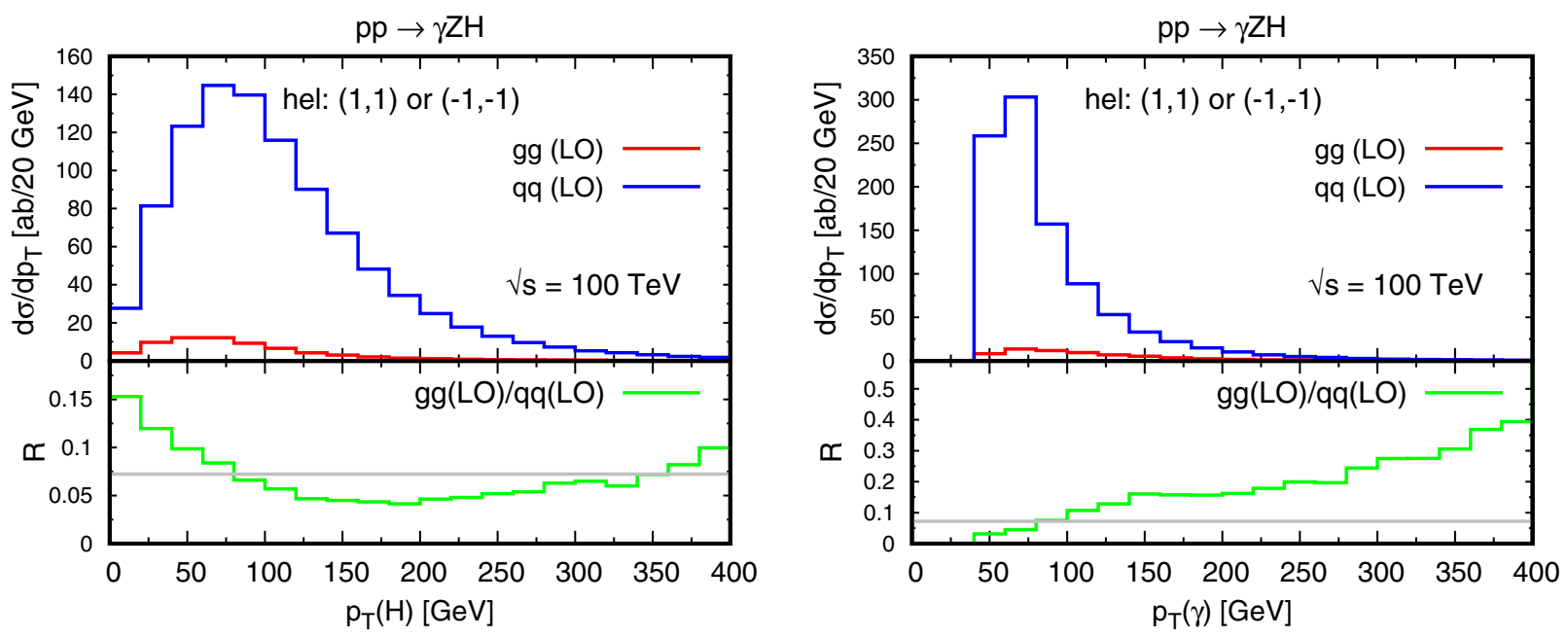

FIG. 7. The kinematic distributions for $g g$ and $q q$ channels when both the $\gamma$ and $Z$ bosons have the same helicity. The ratio of the distributions from the two channels is shown in the lower panel of each figure.

as it is present only in pentagon diagrams whose contribution is small (see Fig. 5). However, it strongly depends on $\kappa_{V}$, as the box-diagrams contribution is much more than the pentagon-diagram contribution. We find that the change in cross section for $\kappa_{t}=1.1(0.9)$ is $5.4 \%(-1.2 \%)$. On the other hand, for $\kappa_{V}=1.1(0.9)$ the cross section changes by $18 \%(15 \%)$. We do not show the effect of anomalous couplings on the distribution. It can be understood qualitatively from Eq. (2) and Fig. 5 in the $g g$ channel. The $q q$ channel is sensitive to $\kappa_{V}$ only. The amplitude has overall linear dependence on $\kappa_{V}$ due to which the effect of anomalous coupling $k_{V}$ is flat for both total and differential cross sections.

\section{Predictions for $p p \rightarrow Z Z H$}

The cross sections for $Z Z H$ production via various channels have been tabulated in Table IV along with the corresponding scale uncertainties. The total cross section for $g g \rightarrow Z Z H$ is significantly larger than that of $g g \rightarrow \gamma Z H$. This increase is mainly due to the contribution from the axial-vector coupling of $Z$ with quarks. The $g g$ channel contributions to $Z Z H$ production at 14,27 , and $100 \mathrm{TeV}$ colliders are 124,579 , and 7408, respectively. The corresponding values of the $\mathrm{LO} q q$ channel contributions are 2184,5997 , and $36830 \mathrm{ab}$, respectively. The ratio, $R_{1}$, is found to be $0.25,0.4$, and 1.05 , respectively. Thus, at

TABLE IV. A comparison of different perturbative orders in QCD coupling contributing to the $p p \rightarrow Z Z H$ cross section at $\sqrt{s}=14,27$, and $100 \mathrm{TeV}$. The ratio $R_{1}$, defined in Eq. (6), quantifies the $g g$ channel contribution with respect to the NLO correction in $q q$ channel process.

\begin{tabular}{lcrrr}
\hline \hline$\sqrt{\mathrm{s}}(\mathrm{TeV})$ & $\sigma_{g g}^{\mathrm{ZZH}, \mathrm{LO}}[\mathrm{ab}]$ & $\sigma_{g g}^{\mathrm{ZZH}, \mathrm{LO}}{ }_{[\mathrm{ab}]}$ & $\sigma_{q q}^{\mathrm{ZZH}, \mathrm{NLO}}{ }_{[\mathrm{ab}]}$ & $R_{1}$ \\
\hline 14 & $124_{-21.0 \%}^{+28.2 \%}$ & $2184_{-0.6 \%}^{+0.2 \%}$ & $2710_{-1.0 \%}^{+1.4 \%}$ & 0.24 \\
27 & $579_{-18.5 \%}^{+23.5 \%}$ & $5997_{-3.4 \%}^{+2.0 \%}$ & $7396_{-1.3 \%}^{+1.3 \%}$ & 0.41 \\
100 & $7408_{-18 \%}^{+22 \%}$ & $36830_{-8.7 \%}^{+8.0 \%}$ & $43940_{-2.6 \%}^{+1.2 \%}$ & 1.04 \\
\hline \hline
\end{tabular}



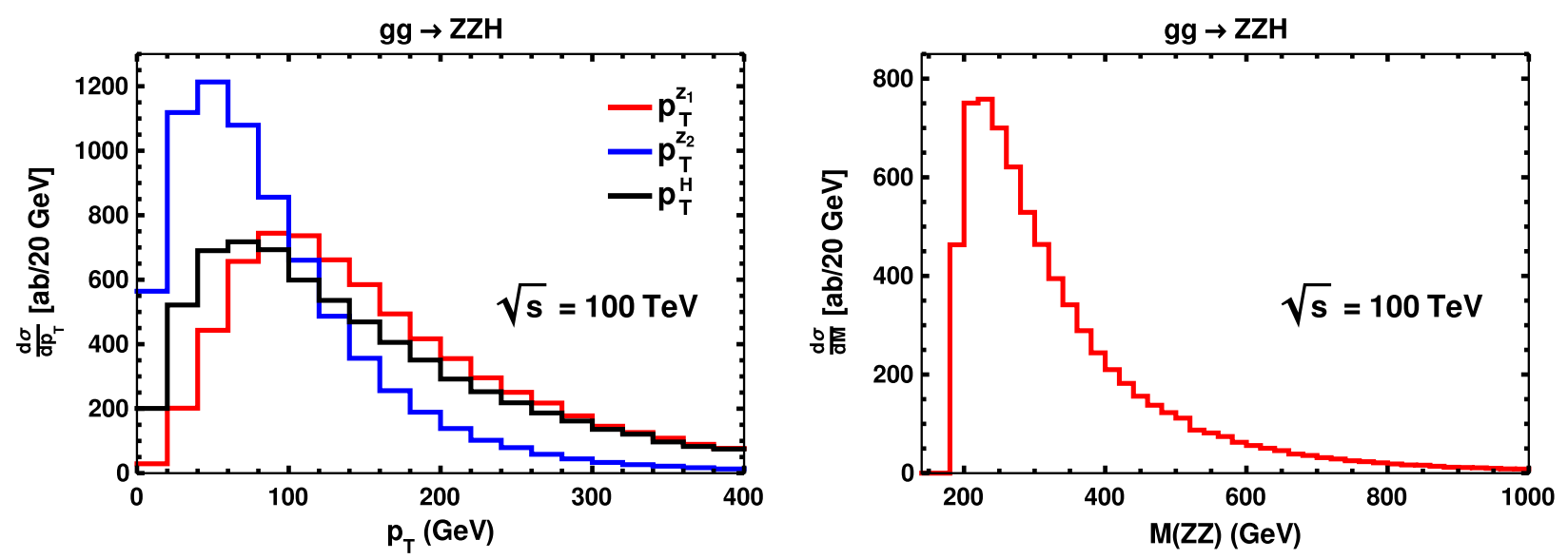

FIG. 8. Kinematic distributions for $g g \rightarrow Z Z H$ in the $\mathrm{SM}$ at the $100 \mathrm{TeV}$ collider. $Z_{1}$ and $Z_{2}$ refer to the hardest and second hardest in $p_{T}$, respectively.

$100 \mathrm{TeV}$, the $g g$ channel contribution is as important as the QCD-NLO correction. As has already been discussed, this increase in ratio $R_{1}$ with collider energy is due to the large gluon flux.

In the $g g$ channel, the scale uncertainties of the total cross sections are in the range of $20 \%-30 \%$ which is similar to the scale uncertainties observed for $\gamma \gamma H$ and $\gamma \mathrm{ZH}$. We find that the uncertainty due to the renormalization scale variation is more than that due to the factorization scale variation. While the change in the renormalization scale mainly changes $\alpha_{s}$, the change in the factorization scale changes the parton distribution function. The uncertainty for the renormalization scale variation is nearly same at all the collider energies. This happens as the contribution to the total cross section comes from nearly the same region of the partonic center-of-mass energy of the process and in every bin of this region, $\alpha_{s}$ changes by nearly the same factor for the change in the renormalization scale. However, uncertainty for the factorization scale variation is different for different colliders. This happens as for different collider energies, different $x$ regions contribute to the process and for different $x$ regions change in parton distribution function with the factorization scale is different, where $x$ is the partonic momentum fraction. We have also observed that with an increase in the factorization scale, for 14 and $27 \mathrm{TeV}$ colliders, the crosssection decreases; however for $100 \mathrm{TeV}$ collider the crosssection increases.

In the tree level $q q$ channel, there is no QCD vertex. So here change in the renormalization scale does not affect the cross section. But, the change in the factorization scale can affect the cross section, and uncertainty increases with collider energy. However, when the NLO-QCD correction is considered, change in either renormalization and factorization scales changes the cross section. The uncertainty in the cross section due to the renormalization scale variation is small as the NLO-QCD correction is much smaller than the LO results. The overall uncertainty in this case is smaller than the LO case, which is expected for higher order calculation.

In Fig. 8, we have plotted $p_{T}$ distributions for leading $p_{T}\left(Z_{1}\right)$, next-to-leading $p_{T}\left(Z_{2}\right)$, and the Higgs boson in the left figure, and the $Z$-pair invariant mass distribution in the right figure for the $100 \mathrm{TeV}$ collider. The $p_{T}$ distributions peak around 100,60 , and $80 \mathrm{GeV}$, respectively. The $M(Z Z)$ distribution peaks around the Z-pair threshold.

Interference of various diagrams plays a major role in $g g \rightarrow \mathrm{ZZH}$ production. In Fig. 9, we have shown the $p_{T}(H)$ distributions for penta, box, triangle, sum of their individual contributions, interference, and total at the $100 \mathrm{TeV}$ collider (FCC-hh). As can be seen, the box diagrams give the largest contribution, then comes the triangle contribution, and the penta contributes the least. Like in the $\gamma Z H$ case, the large box contribution is due to the light quarks in the loop. Further, because of large destructive interference, the total contribution is smaller by about a factor of 5 than the box contribution.

We have found that the top-quark contribution in the $g g Z Z^{*}$-type box diagram is quite significant despite the propagators suppression. This is due to the coupling of the off-shell longitudinal $Z$ boson (effectively the Goldstone boson) with top quark and it is proportional to $m_{t}{ }^{8}$ We show the effect of excluding the top-quark contribution in the $g g Z Z^{*}$-type box diagram [Fig. 1(b)] on the $p_{T}(H)$ distribution in the right panel of Fig. 9. As we expect, excluding the top-quark contribution in the $g g Z Z^{*}$-type box diagram leads to nonunitary behavior in the full amplitude.

In the left figure of Fig. 10, we see that the shape of $p_{T}$ distribution for Higgs boson in the $g g$ and $q q$ channel processes is nearly same at $100 \mathrm{TeV}$ collider (FCC-hh). The relative importance of the $g g$ channel over the $q q$ channel is

\footnotetext{
${ }^{8}$ The results for the $Z Z H$ process presented in the conference proceeding [34] did not include the top-quark contribution. We also fixed a bug in the code, the numerical impact of which has been found to be small.
} 

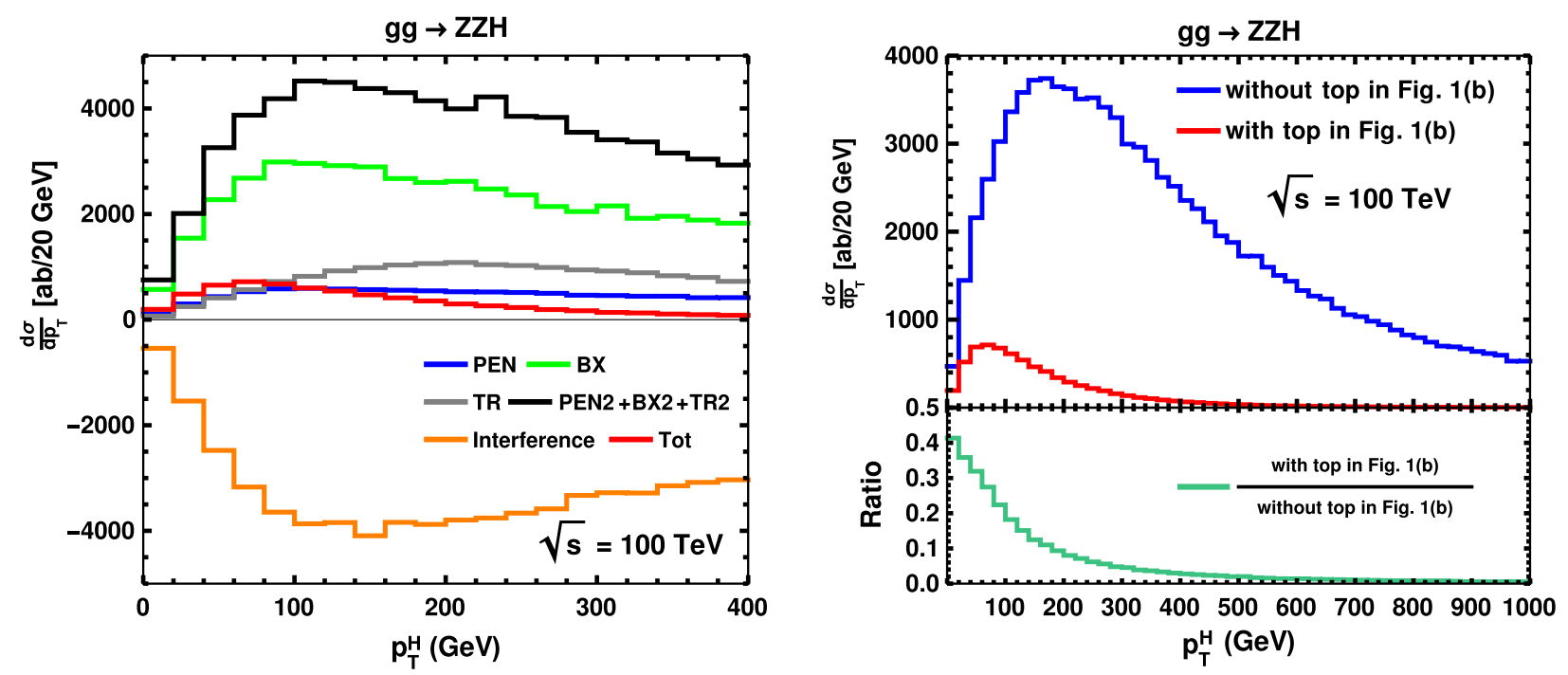

FIG. 9. Left: SM contribution of pentagon (blue), box (green), triangle (gray) diagrams, as well as their squared sum (black), interference (orange), and total (red) contribution to $p_{T}(H)$ distributions in $g g \rightarrow Z Z H$ at 100 TeV collider (FCC-hh). Right: The effect of excluding the top-quark contribution from Fig. 1(b) to full amplitude.
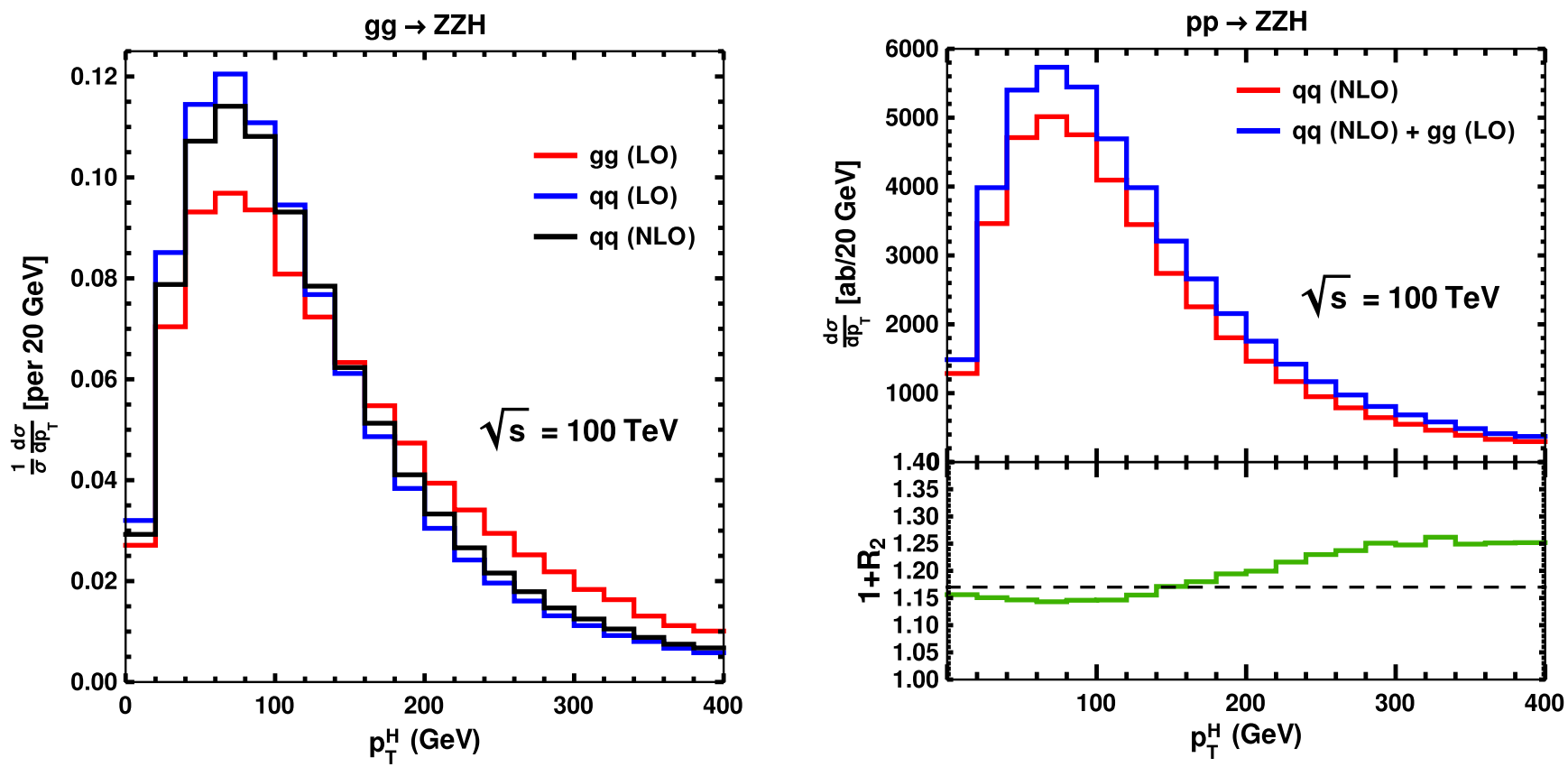

FIG. 10. The left figure shows the normalized distribution for $p_{T}(H)$ in $g g$ and $q q$ channel processes. In the top panel of the right figure, we show the distribution of $q q(\mathrm{NLO})+g g(\mathrm{LO})$ and $q q(\mathrm{NLO})$ production with $p_{T}(H)$. The lower panel shows the ratio of them.

visible in the tail. In the right plot, we give the $p_{T}(H)$ distribution combining $g g$ and $q q$ (NLO) contributions as the best prediction from our calculations. In the bottom panel of the plot, $R_{2}$ signifies the ratio of the differential cross section from the $g g$ channel to that from NLO $q q$ channel process. The dashed line shows the ratio of corresponding total cross sections, which is 0.17 . At the tail of the distribution, we see the $g g$ channel contribution becomes further important, but the differential cross section itself is quite small.

Once again we find that if we categorize events based on the helicity states of the two $Z$ bosons, the relative importance of the $g g$ channel contribution over the $q q$ channel contribution can be increased. From Fig. 11, we 
see that in the $g g$ channel the longitudinal $Z$ bosons contribute the most, while in the $q q$ channel their transverse helicity states give the dominant contribution. The relative cross section of the $g g$ channel with respect to the $q q$ channel is about $20 \%$. However, if we restrict ourselves to the case when both $Z$ bosons are longitudinally polarized, then this ratio almost doubles. Since the cross section for these polarized states for the $g g$ channel is about $2000 \mathrm{ab}$, there will be enough events to observe this process at a $100 \mathrm{TeV}$ machine. At the distribution level, from the Fig. 12, we observe that if we restrict ourselves to the contributions from the longitudinal $Z$ bosons with $p_{T}(H)$ beyond $150 \mathrm{GeV}$, the relative contribution of the $g g$ channel increases significantly. Experimentally, one may look at the signature $l^{+} l^{-} l^{+} l^{-} b \bar{b}$. This signature is obtained when $Z \rightarrow$ $l^{+} l^{-}(l=e / \mu)$ and for $H \rightarrow \bar{b} b$. Taking into account the branching ratios, and $b$-tagging efficiency, one may expect about 75 events at the FCC-hh collider (with $30 \mathrm{ab}^{-1}$ integrated luminosity) from the $g g$ channel and about 210 events from the $q q$ channel. This is when both $Z$ bosons are longitudinally polarized. This number will go down when detection and kinematic-cut efficiency factors are included. However, if in the future, one could use hadronic decay modes of a $Z$ boson to measure its polarization, then the number of events would increase.

As can be seen from Eq. (3), the $g g$ channel depends on $\kappa_{t}, \kappa_{V}$, and $\kappa_{\lambda}$. We vary these $\kappa^{\prime}$ 's by $10 \%$ from their SM values. The $g g$ channel strongly depends on both $\kappa_{t}$ and $\kappa_{V}$. In the $g g$ channel, $\pm 10 \%$ change in $\kappa_{t}$ causes $68 \%$ and $-18 \%$ variations in the cross section, respectively. And $\pm 10 \%$ change in $\kappa_{V}$ causes $45 \%$ and $-28 \%$ changes in the cross section, respectively. A similar variation in $\kappa_{\lambda}$ does
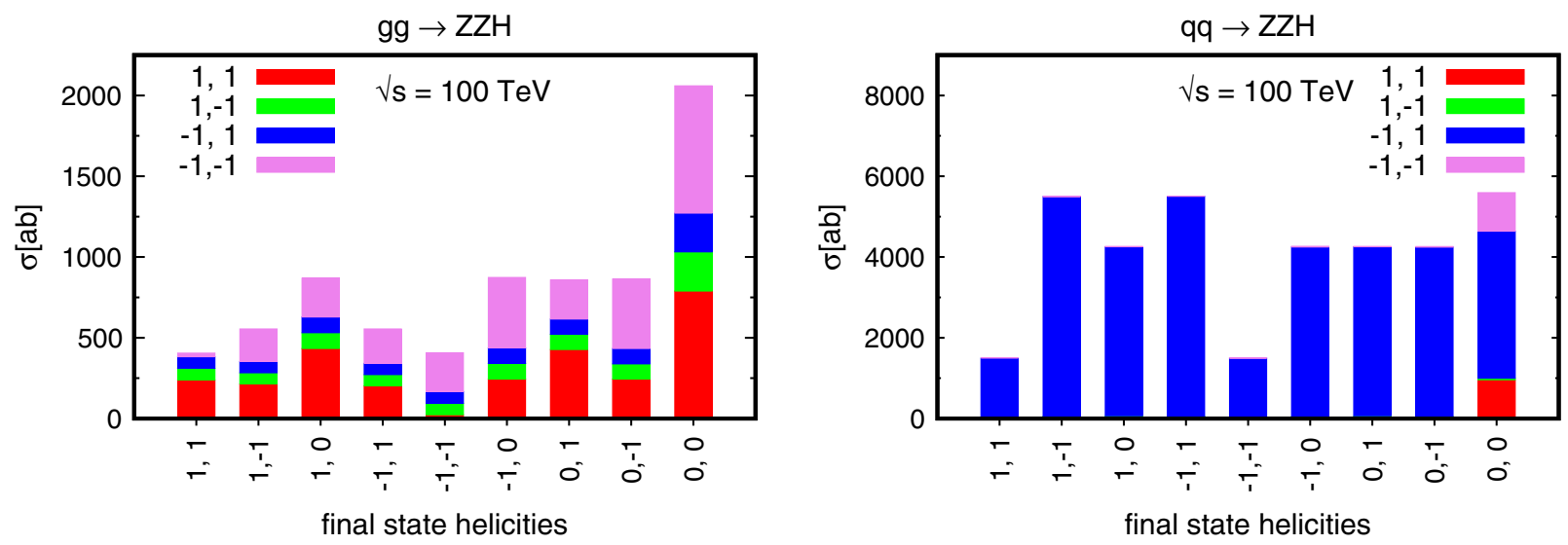

FIG. 11. LO cross section for $Z Z H$ production in different helicity configurations in $g g$ (left) and $q q$ (right) channels. Legends correspond to different helicities of initial states.
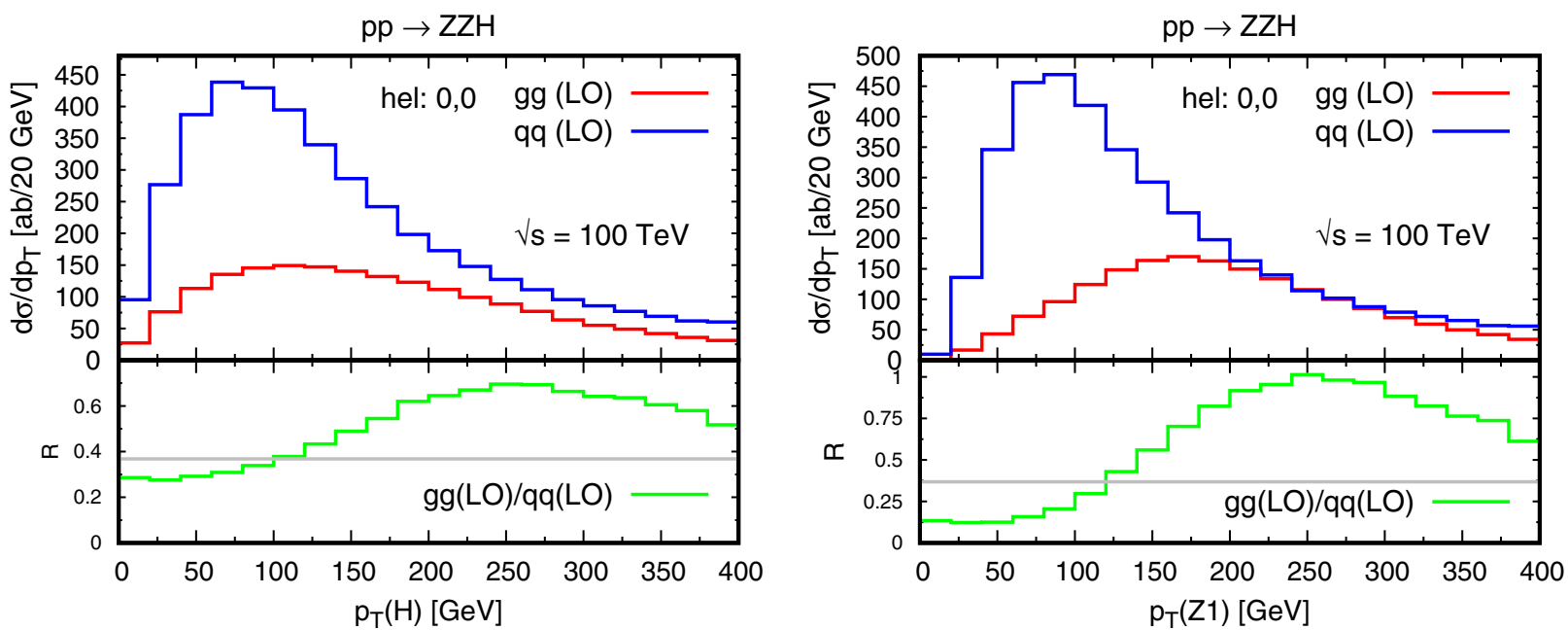

FIG. 12. The kinematic distributions from the $g g$ and $q q$ channels when the final state $Z$ bosons are longitudinal. The ratio of the distributions from the two channels has been shown in the lower panel of each figure. In the right figure, $Z_{1}$ denotes the harder of two $Z$ bosons in $p_{T}$. 

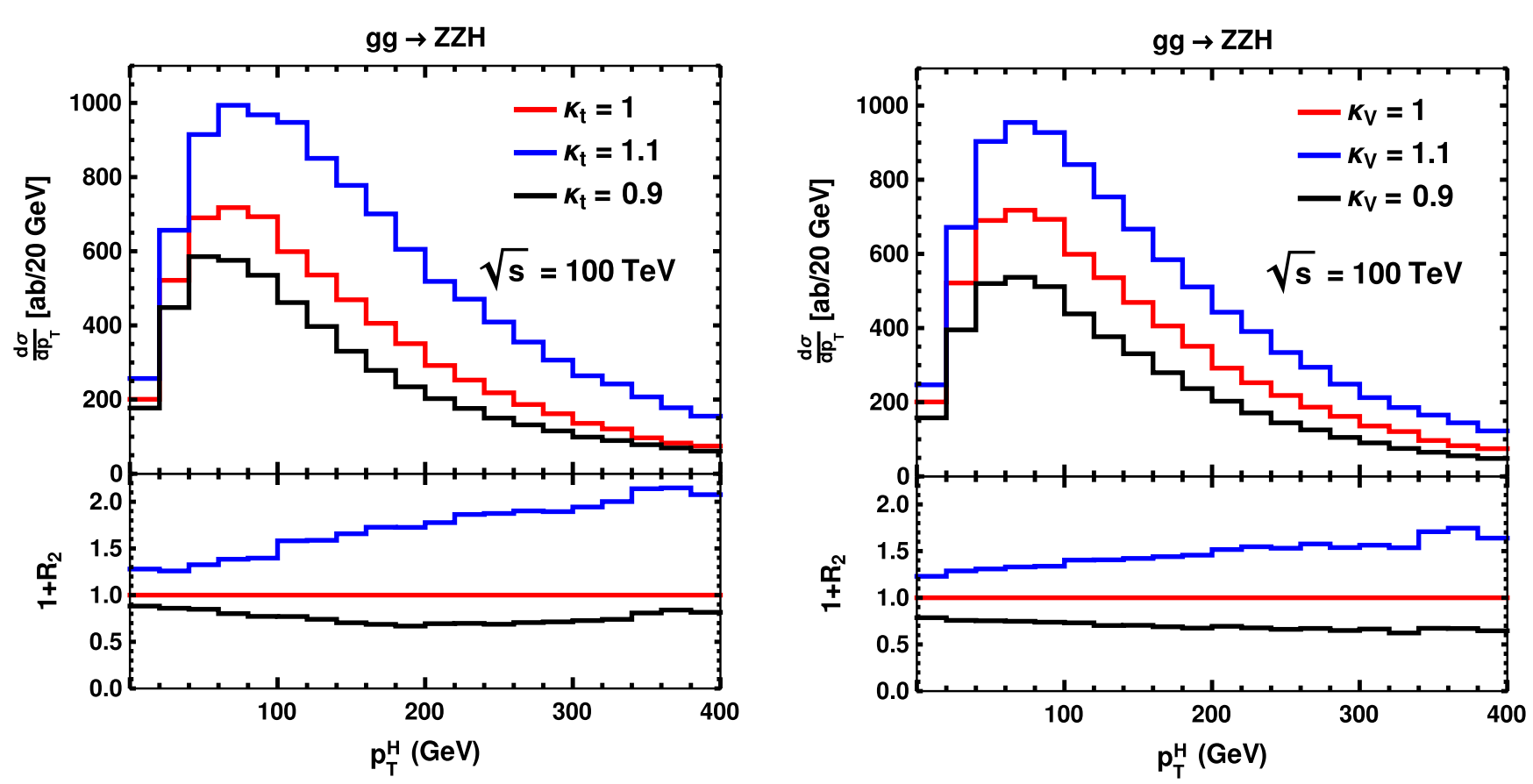

FIG. 13. Effect of anomalous values of $\kappa_{t}$ and $\kappa_{V}$ on $p_{T}(H)$ distribution for $Z Z H$ production via the $g g$ channel. The lower panels display the ratio of BSM and SM distributions.

not lead to much variation in the total cross section. Since this coupling is not yet well constrained, we will discuss it in detail in Sec. V E.

In Fig. 13, we display the effect of $\kappa_{t}$ and $\kappa_{V}$ on $p_{T}(H)$ distribution. We show the absolute distribution in the top panel, while in the bottom panel we show the ratio of distribution with anomalous coupling to that with the SM coupling. We can see that in the presence of anomalous $\kappa_{t}$ and $\kappa_{V}$, the shape of the distribution remains more or less same. However, due to nontrivial interference effects, the modifications in the presence of anomalous couplings are not same in all the bins. We see that for $\kappa_{t}=1.1$ the cross section in the bins near the tail of the distribution increases by a factor of 2 . On the other hand, for $\kappa_{V}=1.1$, the maximum change in the cross section is around 1.5. Thus, the tail of the distributions are more sensitive to modifications in couplings due to high scale new physics. The $q q$ channel depends mainly on $\kappa_{V}$. However, as we have

TABLE V. A comparison of different perturbative orders in QCD coupling contributing to the $p p \rightarrow W W H$ hadronic cross section at $\sqrt{s}=14,27$, and $100 \mathrm{TeV}$. The ratio $R_{1}$ defined in Eq. (6) quantifies the $g g$ channel contribution with respect to the $q q$ (NLO) correction. The $q q$ results are reported in four flavor scheme.

\begin{tabular}{lrrrr}
\hline \hline$\sqrt{\mathrm{s}}(\mathrm{TeV})$ & $\sigma_{q q}^{W W H, \mathrm{LO}}[\mathrm{ab}]$ & $\sigma_{q q}^{W W H, \mathrm{LO}}[\mathrm{ab}]$ & $\sigma_{q q}^{W W H, \mathrm{NLO}}[\mathrm{ab}]$ & $R_{1}$ \\
\hline 14 & $290_{-21.0 \%}^{+27.6 \%}$ & $8658_{-0.7 \%}^{+0.3 \%}$ & $11220_{-1.1 \%}^{+1.5 \%}$ & 0.11 \\
27 & $1344_{-18.8 \%}^{+2.5 \%}$ & $23040_{-2.7 \%}^{+2.1 \%}$ & $30090_{-1.8 \%}^{+1.7 \%}$ & 0.19 \\
100 & $17403_{-17.8 \%}^{+20.6 \%}$ & $128000_{-8.1 \%}^{+7.5 \%}$ & $167300_{-3.3 \%}^{+2.0 \%}$ & 0.44 \\
\hline \hline
\end{tabular}

considered bottom quark contributions also, the $q q$ channel depends on $\kappa_{\lambda}$ as well. In the $q q$ channel, $\kappa_{V}$ comes as an overall factor both for LO and NLO amplitudes, and so the effect of $10 \%$ change in $\kappa_{V}$ causes around 20\% change in the cross section, both at total and differential levels. We find a very mild dependence on $\kappa_{\lambda}$.

\section{Predictions for $p p \rightarrow W W H$}

The cross section for this process is the largest among all the $V V H$ processes considered in this paper. In Table $\mathrm{V}$, we report the cross section predictions for $W W H$ process at different collider center-of-mass energies. The $g g$ channel contributions to $W W H$ production at 14,27 , and $100 \mathrm{TeV}$ colliders are 290, 1344, and $17403 \mathrm{ab}$, respectively. These numbers are roughly 2.3 times higher than $Z Z H$ cross sections. As regards scale uncertainties, the $g g \rightarrow W W H$ cross sections follow the same pattern as observed in $g g \rightarrow Z Z H$. The corresponding values of the LO $q q$ channel cross sections are 8658, 23040, and $128000 \mathrm{ab}$, respectively. ${ }^{9}$ The ratio, $R_{1}$, is found to be $0.15,0.19$, and 0.43 , respectively. Unlike $Z Z H$ production, the contribution of the $g g$ channel is relatively smaller.

In the left figure of Fig. 14, we can see that the $p_{T}$ distribution of $W^{+}$and $W^{-}$overlap with each other, which is expected in the case of the $g g$ channel. The $p_{T}(H)$ distribution peaks around $100 \mathrm{GeV}$, and its fall in the tail is

\footnotetext{
${ }^{9}$ Because of technical reasons in the NLO calculation using MadGraph5_aMC@NLO, the $q q$ results are provided in the four flavor scheme.
} 

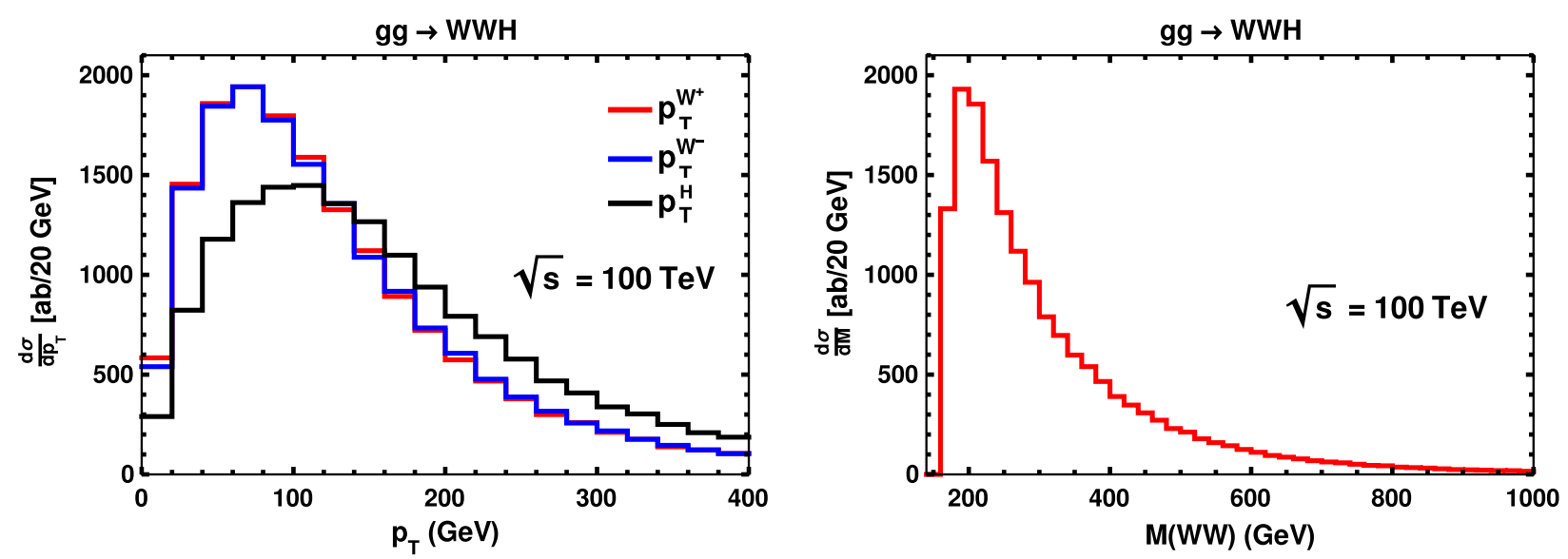

FIG. 14. $\quad p_{T}$ and $M(W W)$ distributions for $g g \rightarrow W W H$ in the SM at the $100 \mathrm{TeV}$ collider (FCC-hh).

slower than that of $p_{T}\left(W^{ \pm}\right)$distributions. In the right figure of Fig. 14, the distribution for invariant masses of $W^{+}$and $W^{-}$has been shown, which peaks around $200 \mathrm{GeV}$.

Like the $g g \rightarrow Z Z H$ production case, in $g g \rightarrow W W H$ production also, interference of various diagrams plays a major role. On the left of Fig. 15, we have shown $p_{T}(H)$ distributions for individual topologies as well as for their interference at a $100 \mathrm{TeV}$ collider. The box contribution is the largest in all the bins while the pentagon contribution is the lowest beyond $p_{T}>100 \mathrm{GeV}$. The total contribution is much smaller than the box contribution because of a strong destructive interference effect which is shown by the orange line in the figure.

Because of the presence of top quark propagators in the $g g W W^{*}$-type box diagram, one may naively think of a suppressed contribution from the third generation quarks at low $p_{T}(H)$. In Fig. 15, we show the effect of excluding the third generation quark contribution from the $g g W W^{*}$ type box diagram, on the $p_{T}(H)$ distribution. Like in $g g \rightarrow \mathrm{ZZH}$, the third generation quark contribution in the $g g W W^{*}$-type box diagram is necessary for the unitarization of the full amplitude.

In the left plot of Fig. 16, the normalized $p_{T}$ distributions for the Higgs boson in the $g g$ and $q q$ channel processes have been shown for the $100 \mathrm{TeV}$ collider (FCC-hh). The $p_{T}(H)$ distribution in the $g g$ channel peaks slightly on the harder side making the channel more relevant in higher $p_{T}(H)$ bins. To quantify it better we also plot the ratio of distributions due to $q q(\mathrm{NLO})+g g$ (LO) and $q q$ (NLO). At the differential level, the ratio varies between 1.05 and 1.18 compared to its value (1.1) for the total cross section. Once again, we find that the $g g$ channel contribution is more relevant at higher $p_{T}$ where its contribution reaches $18 \%$.
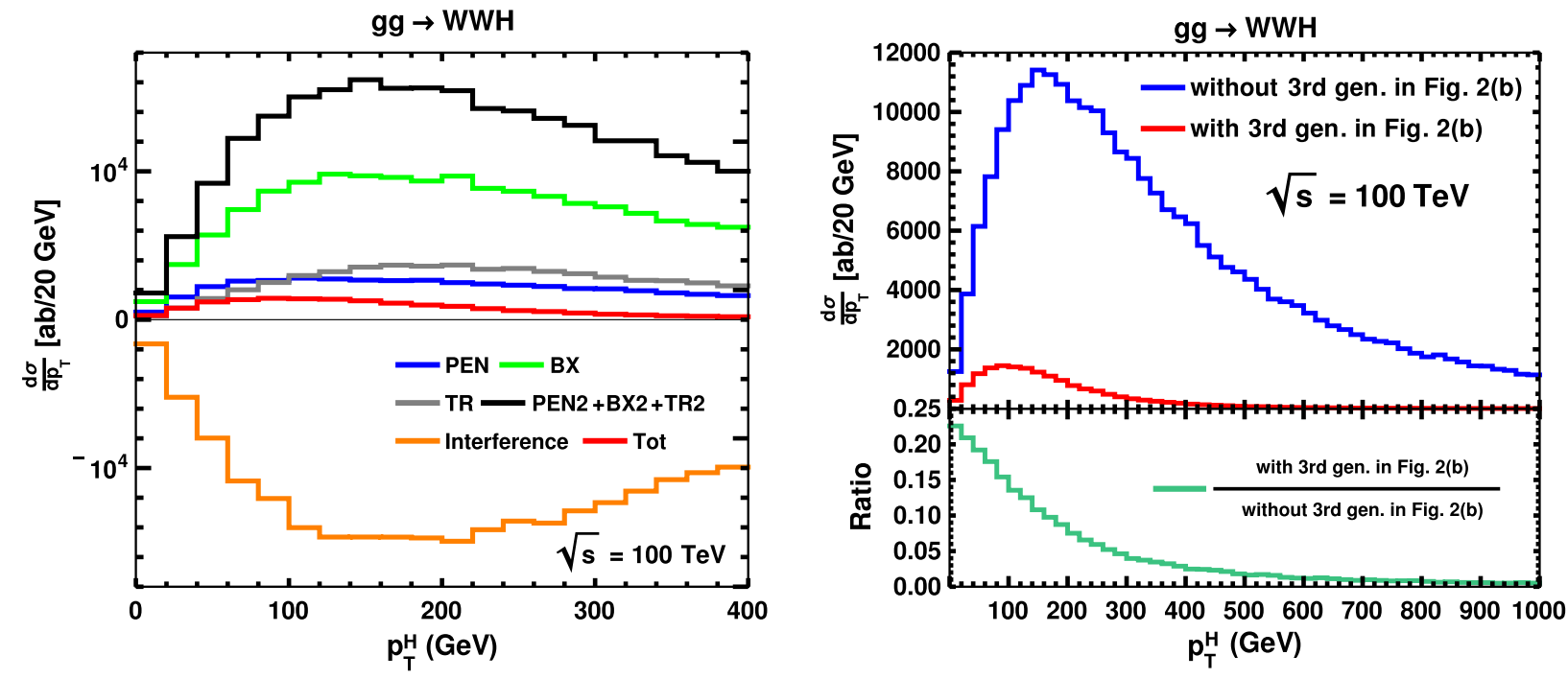

FIG. 15. Left: SM contribution of pentagon (blue), box(green), triangle (gray) diagrams, as well as their square sum, interference, and total contribution to $p_{T}(H)$ distributions in $g g \rightarrow W W H$ at $100 \mathrm{TeV}$ FCC-hh collider. Right: The effect of excluding third generation quark contribution from Fig. 2(b) to full amplitude. 

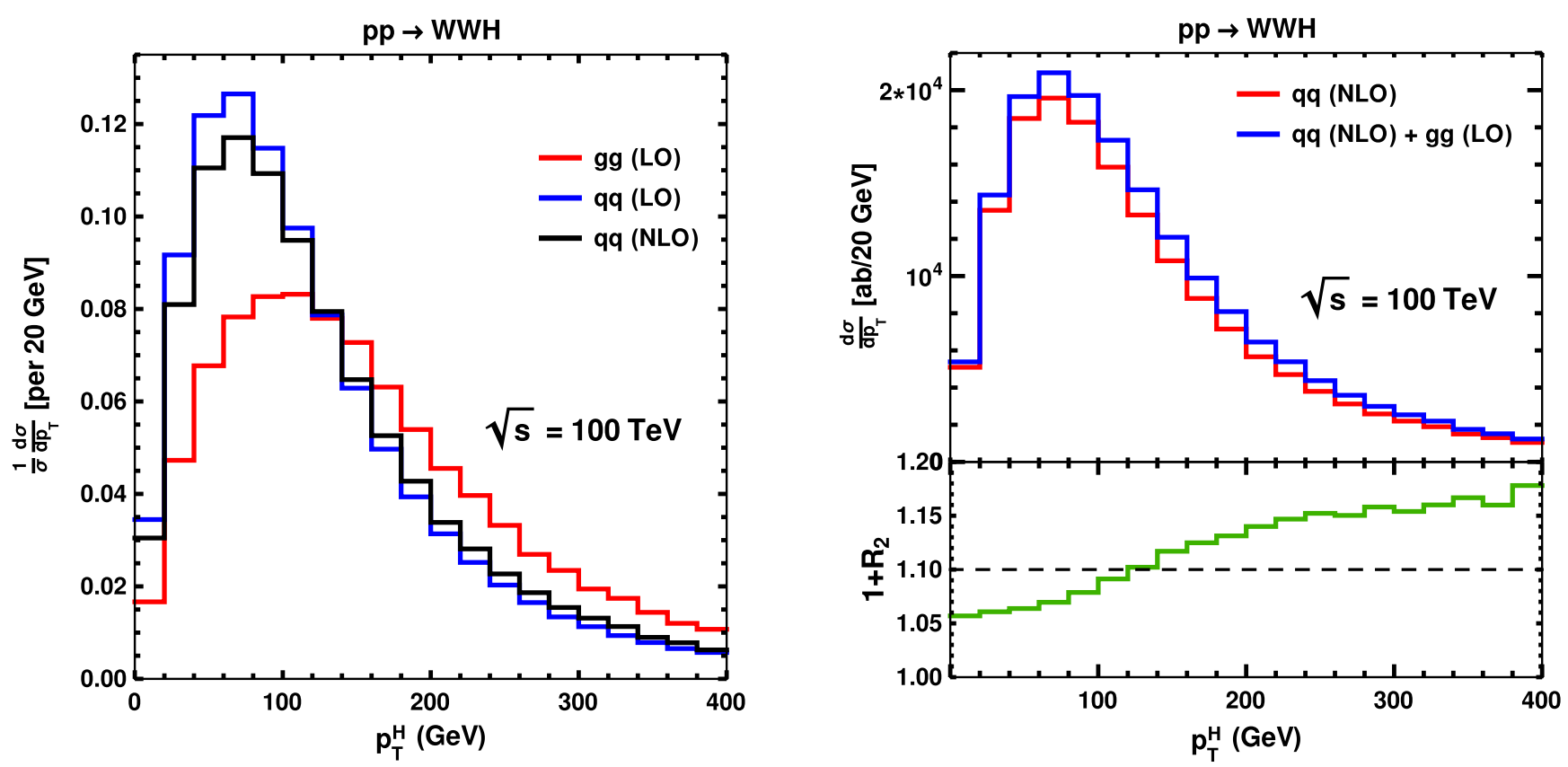

FIG. 16. The left figure shows the normalized distribution for $p_{T}(H)$ in the $g g$ and $q q$ channel processes. In the top panel of the right figure, we show the distribution due to $q q(\mathrm{NLO})+g g(\mathrm{LO})$ and $q q(\mathrm{NLO})$ production with $p_{T}(H)$. The lower panel shows their ratio. Results do not include the contribution of the $b b$ channel process.
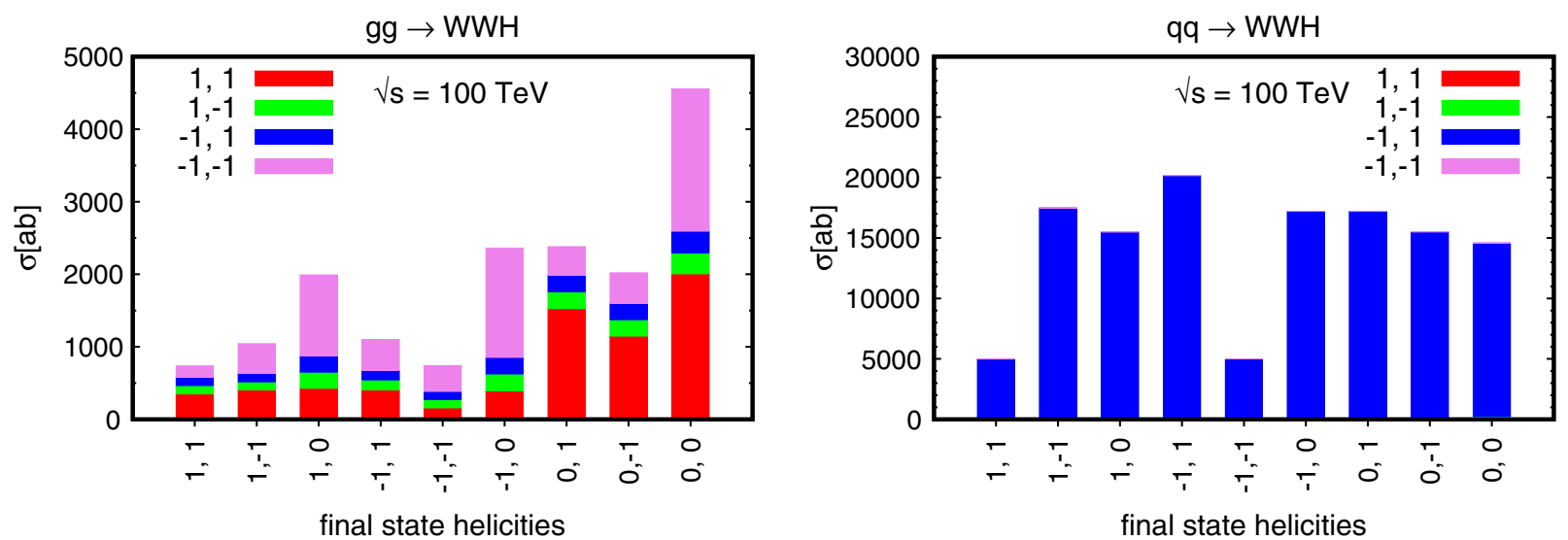

FIG. 17. LO cross section for $W W H$ production in different helicity configurations in $g g$ (left) and $q q$ (right) channels. Legends correspond to different helicities of initial states.

Similar to the case of $Z Z H$, for this process also, the cross section in the $g g$ channel is dominated by longitudinally polarized $W$ bosons (Fig. 17). The relative contribution of this channel is about $13 \%$, with respect to the $q q$ channel. However, when both $W$ bosons are longitudinally polarized, then this ratio increases to $32 \%$. There will also be enough events at a $100 \mathrm{TeV}$ collider to observe the $g g$ channel contribution. The relative contribution of the $g g$ channel over the $q q$ channel can be further increased by requiring the $p_{T}(W)$ to be beyond a certain value between 50 and $100 \mathrm{GeV}$; see Fig. 18. Here also one may consider leptonic decay channel for $W$ bosons, as that will help in the measurement of its polarization. We consider the $l^{+} \nu_{l} l^{-} \bar{\nu}_{l} b \bar{b}$ final state as the signature. Here, as before $l=e / \mu$. In the literature, various techniques, including neural network methods have been discussed to measure the $W$-boson momentum [72]. Taking into account the branching ratios and the $b$-tagging efficiency, one may expect about 1750 events from the $g g$ channel and 5900 events from the $q q$ channel at the FCC-hh collider with $30 \mathrm{ab}^{-1}$ integrated luminosity. The number of these events would change depending on the detector and kinematic-cut efficiency factors.

Next, we focus on the effect of anomalous couplings on the total and differential cross sections. The $g g$ channel depends on $\kappa_{t}, \kappa_{\lambda}$, and $\kappa_{V}$ [see Eq. (4)]. We find that the 

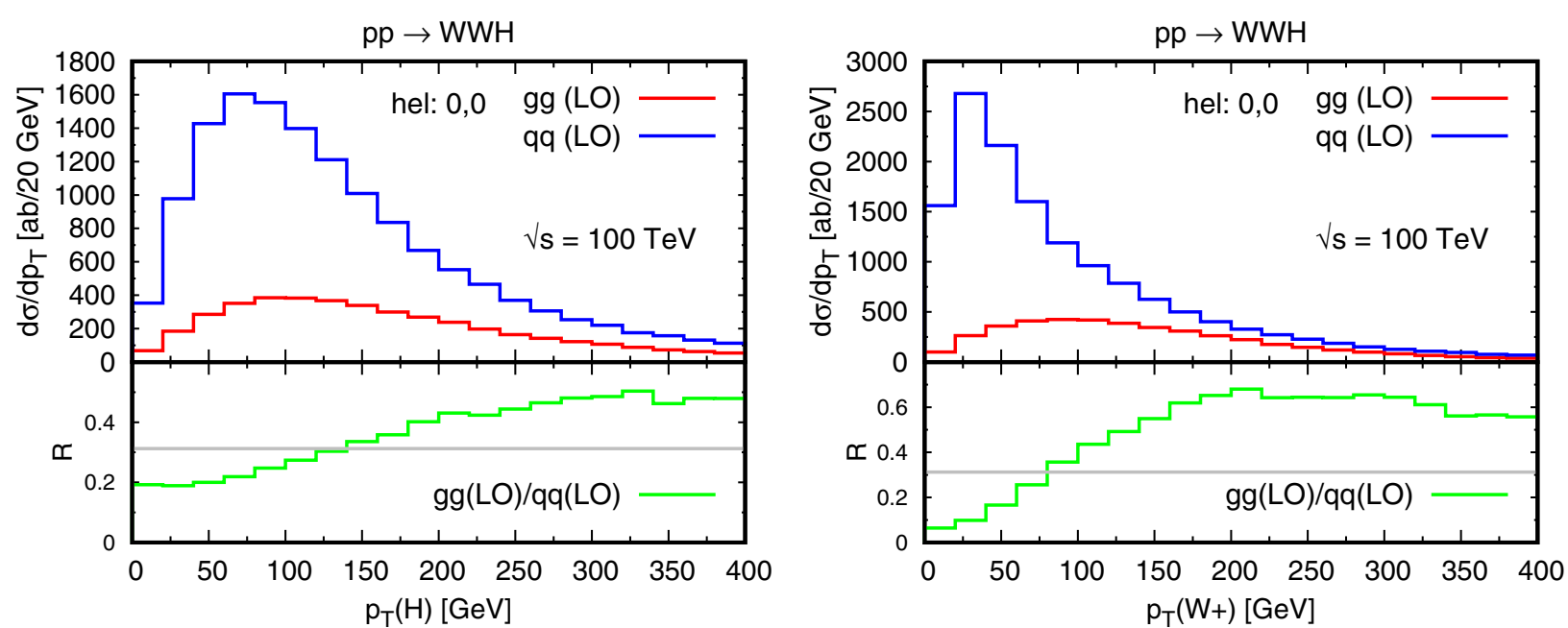

FIG. 18. Comparing the $g g$ and $q q$ channel contributions to $W^{+} W^{-} H$ for events with longitudinal $W$ bosons.

channel is mostly sensitive to $\kappa_{V}$ and $\kappa_{t}$. For $\kappa_{V}=1.1(0.9)$ the cross section changes by about $38 \%(-26 \%)$. While, for $\kappa_{t}=1.1(0.9)$ the cross section changes by about $54 \%$ $(-3 \%)$. The dependence on $\kappa_{\lambda}$ is found to be relatively small. In Fig. 19, we show the effect of $\kappa_{t}$ and $\kappa_{V}$ on the $p_{T}(H)$ distribution for the $g g$ channel. We do not show the distribution for anomalous $\kappa_{\lambda}$ as its effect on the cross section is very small for $10 \%$ variation. We see that the shape remains more or less the same in the presence of anomalous couplings. We see that in the bins around $400 \mathrm{GeV}$, this ratio is around 1.5 for $\kappa_{t}=1.1$ and $\kappa_{V}=1.1$. For $\kappa_{t}=0.9$, the ratio remains close to 1 throughout all the bins and for $\kappa_{V}=0.9$, it is in the range $0.7-0.8$. Similar to the case for $q q \rightarrow Z Z H$, the $q q \rightarrow W W H$ cross section is also

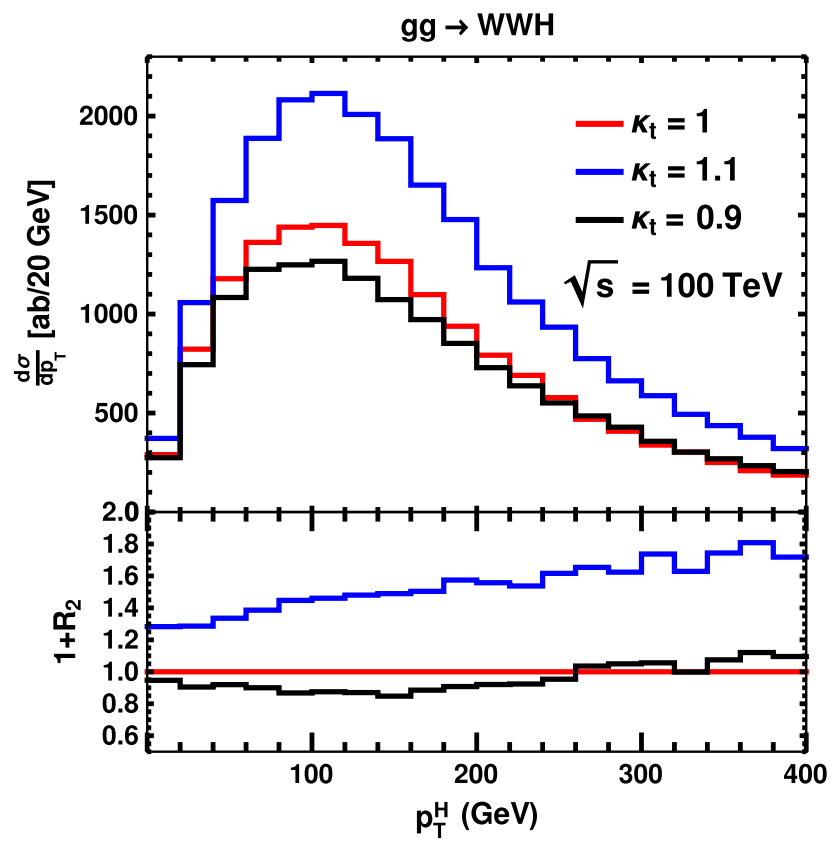

proportional to $\kappa_{V}^{2}$ at LO and NLO(QCD). So here as well, a $10 \%$ change in $\kappa_{V}$ gives around a $20 \%$ obvious change in the cross section, both at the total and differential levels.

\section{E. Remarks on anomalous $\mathrm{HHH}$ and $H H V V$ couplings}

We have seen that the gluon fusion $Z Z H$ and $W W H$ processes are most relevant for BSM physics due to their large cross sections. We found that their cross sections do not change much for a $10 \%$ variation in $\kappa_{\lambda}$. However, we know that this coupling is presently unconstrained by the experimental data. According to the future projections for HL-LHC, only values $\kappa_{\lambda} \lesssim-2$ and $\kappa_{\lambda} \gtrsim 8$ can be ruled out



FIG. 19. Effect of anomalous values of $\kappa_{t}$ and $\kappa_{V}$ on $W W H$ production via the $g g$ channel. The upper panel shows absolute distribution, and the lower panel shows the ratio of the BSM and SM distributions. 

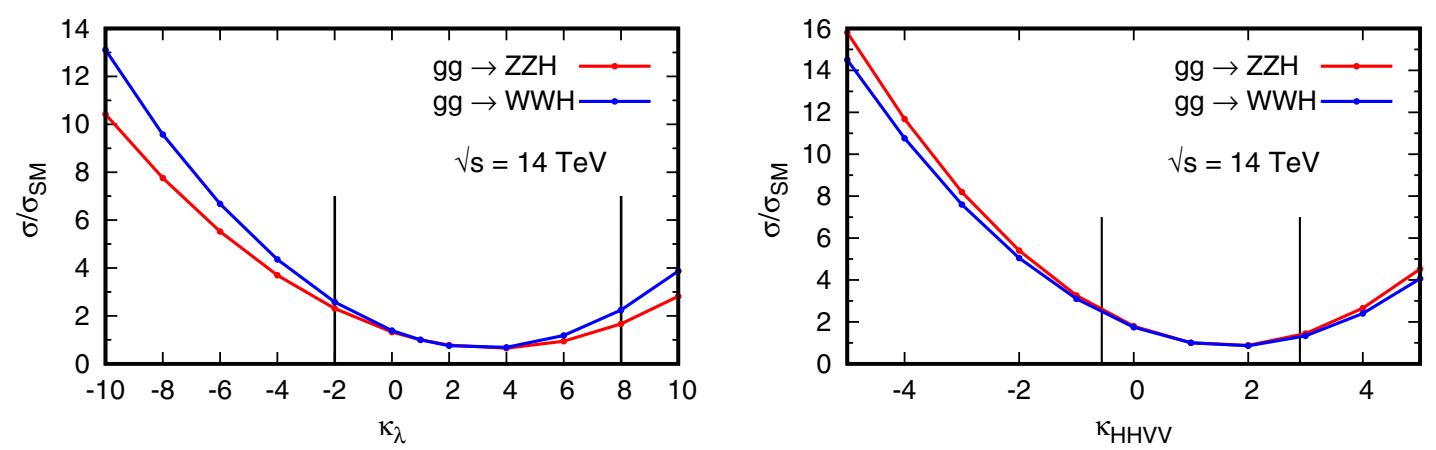

FIG. 20. Dependence of $g g \rightarrow Z Z H, W W H$ cross sections on $H H H$ (left) and $H H V V$ (right) couplings at $14 \mathrm{TeV}$. The vertical lines in the left plot represent projected sensitivity on $\kappa_{\lambda}$ at HL-LHC and those on the right represent current sensitivity on $\kappa_{H H V V}$ at the LHC.
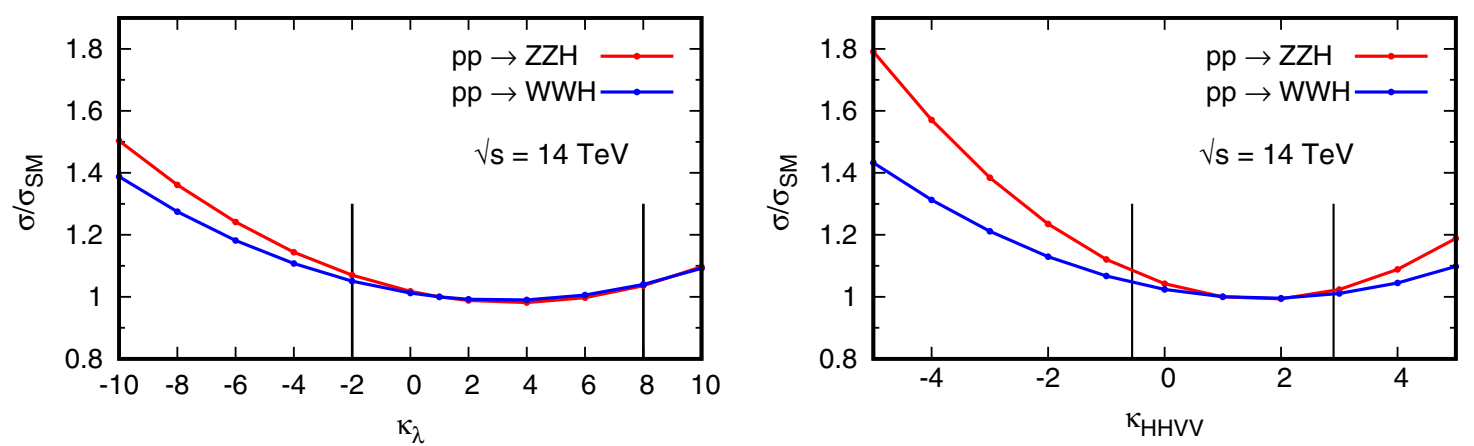

FIG. 21. Dependence of $p p \rightarrow Z Z H, W W H$ cross sections on $H H H$ (left) and $H H V V$ (right) couplings at 14 TeV. The vertical lines in the left plot represent projected sensitivity on $\kappa_{\lambda}$ at HL-LHC and those on the right represent current sensitivity on $\kappa_{H H V V}$ at the LHC.

[9]. In this range the cross section for $\mathrm{ZZH}$ and $W W H$ processes in the $g g$ channel varies significantly. In fact, it can change maximum by a factor of 3 . This is shown in the left panel of Fig. 20. Notice that the $W W H$ process is more affected by the anomalous $H H H$ coupling than the $Z Z H$ process.

Although in the SM model the $\operatorname{HHVV}(V=Z, W)$ coupling is correlated to the $H V V$ coupling, in the presence of new physics this correlation may not exist. Keeping this possibility in mind, we have varied the $H H V V$ coupling independently ${ }^{10}$ and we find that the cross section changes very strongly. This is shown in the right panel of Fig. 20. We can see that the effect of the $H H V V$ coupling is relatively larger on $g g \rightarrow Z Z H$ than on $g g \rightarrow W W H$. Close to $\mathrm{SM}$ values, the difference is negligible. According to a recent search for Higgs boson pair production via vectorboson fusion carried out by the ATLAS Collaboration using $126 \mathrm{fb}^{-1}$ data collected at $13 \mathrm{TeV}$ LHC, the allowed values of $\kappa_{H H V V}$ lie in the range $(-0.56,2.89)$ at $95 \%$ confidence level [16].

The quantity plotted in Fig. 20 is known as signal strength $(\mu)$ which has been utilized by experimentalists as

\footnotetext{
${ }^{10}$ It should be noted that independent variations of $H V V$ and $H H V V$ couplings can be done systematically in an effective field theory framework which is beyond the scope of the present work.
}

observable for data analyses. The signal strength for each process can be parametrized as

$$
\mu=\frac{\sigma^{\mathrm{BSM}}}{\sigma^{\mathrm{SM}}}=1+c_{1}^{i}\left(\kappa_{i}-1\right)+c_{2}^{i}\left(\kappa_{i}-1\right)^{2},
$$

where $\kappa_{i}=\kappa_{\lambda}, \kappa_{H H V V}$. In Table VI, we have provided the values of $c_{1}^{i}$ and $c_{2}^{i}$ for $Z Z H$ and $W W H$ processes for the $14 \mathrm{TeV}$ LHC and a $100 \mathrm{TeV}$ pp collider. We note that $c_{2}^{k_{\lambda}}$ is smaller by an order of magnitude than $c_{1}^{k_{\lambda}}$, suggesting a strong interference effect mentioned before. Therefore, $c_{2}^{\kappa_{\lambda}}$ is relevant mostly for large values of $\kappa_{i}$. On the other hand, $c_{2}^{\kappa_{H H V V}}$ is of the same order as $c_{1}^{\kappa_{H H V V}}$. Since $c_{1}^{i}$ is negative, the cross section increase observed in the figures for $\kappa_{i}<1$

TABLE VI. $\quad c_{1}^{i}$ and $c_{2}^{i}$ that appear in the definition of signal strengths for $g g \rightarrow Z Z H, W W H$ processes at the $14 \mathrm{TeV}$ LHC and $100 \mathrm{TeV}$ colliders.

\begin{tabular}{lccccc}
\hline \hline Collider & $g g$ process & $c_{1}^{\kappa_{\lambda}}$ & $c_{2}^{\kappa_{\lambda}}$ & $c_{1}^{\kappa_{H H V V}}$ & $c_{2}^{\kappa_{H H V V}}$ \\
\hline $14 \mathrm{TeV}$ & $Z Z H$ & -0.275 & 0.053 & -0.458 & 0.335 \\
& $W W H$ & -0.318 & 0.071 & -0.440 & 0.301 \\
$100 \mathrm{TeV}$ & $Z Z H$ & -0.256 & 0.046 & -0.563 & 0.772 \\
& $W W H$ & -0.281 & 0.057 & -0.524 & 0.672 \\
\hline \hline
\end{tabular}


is quite significant, which causes the (negative) lower bound on $\kappa$ to be tighter than the (positive) upper one. At a $100 \mathrm{TeV}$ pp collider, while the other $c_{i}$ 's remain more or less same as that in $14 \mathrm{TeV}$ collider, $c_{2}^{\kappa_{H H V V}}$ increases by around a factor of 2, implying the possibility of a far more stringent bound on the $H H V V$ couplings.

Since the $g g$ fusion channel contribution to $\mathrm{ZZH}$ and $W W H$ processes cannot be fully separated from the corresponding contributions from the $q q$ channel, the above result should be interpreted carefully. A realistic estimate of the BSM effects discussed above must include the contributions from the $q q$ channel. Since $q q$ channel contributions are insensitive to $\kappa_{\lambda}$ and $\kappa_{H H V V}$, they can be seen as one of the major backgrounds to the gluon fusion processes. As we have pointed out, the measurement of the polarization of the $W / Z$ boson can help in reducing this background. A systematic signal-background analysis is beyond the scope of the present work. For the benefit of the reader, in Fig. 21, we present the ratio $\sigma / \sigma_{\mathrm{SM}}$ for $p p \rightarrow Z Z H, W W H$ which includes both $q q$ and $g g$ channel contributions as functions of $\kappa_{\lambda}$ and $\kappa_{H H V V}$. In obtaining these results, only standard cuts mentioned in the previous sections have been applied. We can see that at the $14 \mathrm{TeV}$, the ratio of BSM and SM cross sections due to $q q+g g$ channels is significantly smaller than that due to the $g g$ channel alone. Moreover, the $\mathrm{ZZH}$ process turns out to be more affected by $\kappa_{\lambda}$ and $\kappa_{H H V V}$ than the $W W H$.

To be more precise, we find that by changing $\kappa_{\lambda}$ in the range $(-2,8)$, the cross section for $Z Z H$ process changes in the range $7 \%-4 \%$ at the $14 \mathrm{TeV}$. The corresponding change at the $100 \mathrm{TeV}$ falls in the range of $20 \%-8 \%$. On the other hand, when changing $\kappa_{H H V V}$ in the range $(-0.56,2.89)$, the maximum cross section change in $Z Z H$ process is found to be $\sim 8 \%$ and $\sim 46 \%$ at the 14 and the $100 \mathrm{TeV}$, respectively. Again, we may mention that the polarization measurements would increase the fraction of $g g$ channel events, thus increasing the dependence on $\kappa_{H H V V}$.

\section{CONCLUSIONS}

In this paper, we have considered production of $V V^{\prime} H$ $(\gamma \gamma H, \gamma Z H, Z Z H$, and $W W H)$ at proton-proton colliders. We investigated the sensitivity of these processes to various couplings of the Higgs boson, in particular to $\mathrm{HHH}$ and $H H V V$ couplings which are practically unconstrained. Our main focus was the $g g$ channel contribution, which occurs at NNLO in $\alpha_{s}$. The scale uncertainties on the total cross sections are found to be of the order of $20 \%$. A number of checks like UV and IR finiteness and gauge invariance of the amplitudes with respect to the gluons have been performed to ensure the correctness of the calculation. At a $100 \mathrm{TeV}$ collider, the cross sections for these processes via the $g g$ channel range from 0.2 to $17 \mathrm{fb}$, with $g g \rightarrow$ $W W H$ being the dominant channel among all. We have seen that the $g g \rightarrow \gamma \gamma H$ and $g g \rightarrow \gamma Z H$ processes are insignificant background to $g g \rightarrow H H \rightarrow \gamma \gamma H$ and $g g \rightarrow H H \rightarrow \gamma Z H$, respectively.

We have also compared the $g g$ channel contribution with the fixed order NLO-QCD correction to $p p \rightarrow V V^{\prime} H$ in order to emphasize their relative importance. For $\gamma \gamma H$ production, the $g g$ channel can be said to be the only production channel, as the $b b$ channel process contribution is negligibly small. At a $100 \mathrm{TeV}$ collider, the $g g \rightarrow \gamma Z H$ channel contribution is around $6 \%$ of the NLO-QCD correction in the $q q$ channel. The $\gamma Z H$ production shows one interesting feature: with an increase in the $p_{T}$ cut on photon, the $q q$ channel contribution decreases faster than the $g g$ channel contribution. At this collider, the contribution of the $g g$ channel to $Z Z H$ production is as important as the fixed order QCD-NLO correction to the $q q$ channel. On the other hand, the $g g \rightarrow W W H$ channel cross section is around half the fixed order NLO-QCD correction to the $q q$ channel. We have observed strong destructive interference effects among various classes of diagrams in $g g \rightarrow \gamma Z H, Z Z H, W W H$. Besides total cross sections at the LHC, HE-LHC, and FCC-hh, we have obtained relevant kinematic distributions at FCC-hh in the $g g$ channel. We find that the $p_{T}(H)$ spectrum from the $g g$ channel is harder than that from the $q q$ channel for $Z Z H$ and $W W H$ productions. We have also shown that by selecting events based on the polarization of final state vector bosons, the relative contribution of the $g g$ channel over the $q q$ channel can be enhanced.

In addition to the SM results, the effect of anomalous couplings $\left(\kappa_{t}, \kappa_{V}\right.$, and $\left.\kappa_{\lambda}\right)$ for $H t \bar{t}, H V V, H H V V$, and $H H H$ vertices have been studied in the kappa framework. We find that the new physics effects are quite important in $g g \rightarrow$ $\mathrm{ZZH}, W W H$ processes due to nontrivial interference effects in these processes. A $10 \%$ change in $\kappa_{t}$ on the higher side can enhance the $g g \rightarrow Z Z H$ and $W W H$ cross sections by $68 \%$ and $54 \%$, respectively. A similar change in $\kappa_{V}$ enhances these cross sections by about $40 \%$. Unlike in $q q$ channels, the kinematic distributions in $g g$ channels display nontrivial changes in the presence of new physics. The dependence of the $g g$ channel on the $\kappa_{V}$ is stronger than that of the $q q$ channel. By considering events with longitudinally polarized vector bosons for the processes $p p \rightarrow Z Z H, W W H$, we can enhance the fraction of the $g g$ channel contribution. This event sample will have even stronger dependence on $\kappa_{V}$. Since the $H H H$ and $H H V V$ couplings are not well constrained, we have also considered larger independent variations in $\kappa_{\lambda}$ and $\kappa_{H H V V}$. We find that the effect of $\kappa_{H H V V}$ on the cross section is much stronger than that of $\kappa_{\lambda}$. Therefore the process $p p \rightarrow Z Z H, W W H$ with longitudinally polarized $Z$ and $W$ bosons can help in determining the $H H V V$ coupling.

\section{ACKNOWLEDGMENTS}

D. S. would like to acknowledge the use of HPC cluster facility, SAMKHYA, in Institute of Physics, Bhubaneswar. 
A. S. would like to acknowledge fruitful discussions with Xiaoran Zhao.

\section{APPENDIX: COMMENT ON $Z$ MEDIATED TRIANGLE DIAGRAMS IN $g g \rightarrow W W H$}

It is a well-known theorem due to Landau and Yang that a massive spin-one particle cannot decay into two on-shell spin-one massless particles [73,74]. The same theorem can be applied to argue that the $g g \rightarrow Z$ amplitude vanishes for the on-shell $Z$ boson. This can be easily verified at LO using the on-shell conditions for the gluons and the $Z$ boson. In the past, we have shown that even if the $Z$ boson is off-shell, the LO $g g \rightarrow Z^{*}$ can vanish provided the offshell $Z$ boson is linked to a conserved current [23]. This is so because $\mathcal{M}^{\mu \nu \rho}\left(g g \rightarrow Z^{*}\right) \propto p_{Z^{*}}^{\rho}$. This result is useful for many $g g$ channel processes which receive contributions from such triangle topology. $g g \rightarrow W W$ is one such example [75,76]. In our case, $g g \rightarrow W W H$ is the process which depends on $Z$ mediated triangle diagrams. See Figs. 2(h) and 2(i). We will explicitly show that Fig. 2(i)

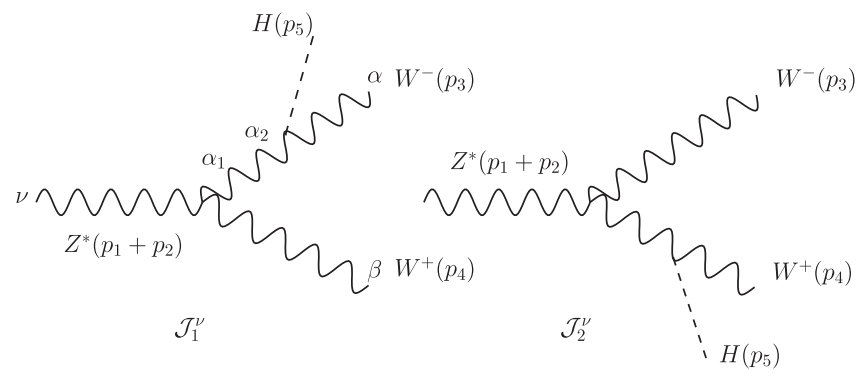

FIG. 22. Currents attached to $Z^{*}$ in Fig. 2(i). All the momenta are incoming.

does not contribute to the $g g \rightarrow W W H$ amplitude. For this we need to just prove that the sum of the currents shown in Fig. 22 when contracted with the momentum $\left(p_{1}+p_{2}\right)^{\nu}$ vanishes.

In the following derivation we use $p_{1}+p_{2}=p_{12}$, $p_{3}+p_{5}=p_{35}$, and $p_{4}+p_{5}=p_{45}$. The polarization vectors of $W^{-}$and $W^{+}$are denoted by $e_{3}^{\alpha}\left(p_{3}\right)$ and $e_{4}^{\beta}\left(p_{4}\right)$, respectively. We first calculate the contraction of current $\mathcal{J}_{1}$ with $p_{12}$.

$$
\begin{aligned}
\mathcal{M}_{1}=p_{12}^{\nu} \mathcal{J}_{1 \nu}= & p_{12}^{\nu}\left(g_{\nu \alpha_{1}}\left(p_{12}-p_{35}\right)_{\beta}+g_{\alpha_{1} \beta}\left(p_{35}-p_{4}\right)_{\nu}+g_{\beta \nu}\left(p_{4}-p_{12}\right)_{\alpha_{1}}\right) \frac{-g^{\alpha_{1} \alpha_{2}}+p_{35}^{\alpha_{1}} p_{35}^{\alpha_{2}} / M_{W}^{2}}{p_{35}^{2}-M_{W}^{2}} g_{\alpha_{2} \alpha} e_{3}^{\alpha}\left(p_{3}\right) e_{4}^{\beta}\left(p_{4}\right) \\
= & \left(p_{12 \alpha_{1}}\left(p_{12}-p_{35}\right) \cdot e_{4}+e_{4 \alpha_{1}} p_{12} \cdot\left(p_{35}-p_{4}\right)+p_{12} \cdot e_{4}\left(p_{4}-p_{12}\right) \alpha_{1}\right) \frac{-e_{3}^{\alpha_{1}}+p_{35}^{\alpha_{1}} p_{35} \cdot e_{3} / M_{W}^{2}}{p_{35}^{2}-M_{W}^{2}} \\
= & \left(-p_{12} \cdot e_{3}\left(p_{12}-p_{35}\right) \cdot e_{4}-e_{3} \cdot e_{4} p_{12} \cdot\left(p_{35}-p_{4}\right)-p_{12} \cdot e_{4}\left(p_{4}-p_{12}\right) \cdot e_{3}\right) /\left(p_{35}^{2}-M_{W}^{2}\right) \\
& +\frac{p_{35} \cdot e_{3}}{M_{W}^{2}\left(p_{35}^{2}-M_{W}^{2}\right)}\left(p_{12} \cdot p_{35}\left(p_{12}-p_{35}\right) \cdot e_{4}+p_{35} \cdot e_{4} p_{12} \cdot\left(p_{35}-p_{4}\right)-p_{12} \cdot e_{4}\left(p_{4}-p_{12}\right) \cdot p_{35}\right) .
\end{aligned}
$$

Using momentum conservation $p_{12}=-p_{35}-p_{4}$ and transversality conditions $e_{3} \cdot p_{3}=e_{4} \cdot p_{4}=0$, we get

$$
\begin{aligned}
\mathcal{M}_{1}= & \left(-2 p_{35} \cdot e_{4} p_{45} \cdot e_{3}+e_{3} \cdot e_{4}\left(p_{35}^{2}-p_{4}^{2}\right)+p_{35} \cdot e_{4}\left(p_{4}+p_{45}\right) \cdot e_{3}\right) /\left(p_{35}^{2}-M_{W}^{2}\right) \\
& +\frac{p_{35} \cdot e_{3}}{M_{W}^{2}\left(p_{35}^{2}-M_{W}^{2}\right)}\left(2 p_{35} \cdot e_{4}\left(p_{35}+p_{4}\right) \cdot p_{35}-p_{35} \cdot e_{4}\left(p_{35}^{2}-p_{4}^{2}\right)-p_{35} \cdot e_{4}\left(2 p_{4}+p_{35}\right) \cdot p_{35}\right) \\
= & \frac{-p_{35} \cdot e_{3} p_{35} \cdot e_{4}+e_{3} \cdot e_{4}\left(p_{35}^{2}-p_{4}^{2}\right)}{\left(p_{35}^{2}-M_{W}^{2}\right)}+\frac{p_{35} \cdot e_{3}}{M_{W}^{2}\left(p_{35}^{2}-M_{W}^{2}\right)} p_{35} \cdot e_{4} p_{4}^{2} .
\end{aligned}
$$

Using on-shell condition $p_{4}^{2}=M_{W}^{2}$, we arrive at

$$
\mathcal{M}_{1}=e_{3} . e_{4}
$$

Following similar steps, it can be shown that the contraction of current $\mathcal{J}_{2}$ with $p_{12}$ leads to

$$
\mathcal{M}_{2}=p_{12}^{\nu} \mathcal{J}_{2 \nu}=-e_{3} \cdot e_{4} .
$$

Combining Eqs. (A6) and (A7) we obtain the desired result: $\mathcal{M}_{1}+\mathcal{M}_{2}=0$. Thus we have proved that indeed the current associated with $Z^{*}$ in Fig. 22 is a conserved current and therefore the triangle amplitude for Fig. 2(i) vanishes for each quark flavor in the loop. It can be verified explicitly that the current associated with $Z^{*}$ in Fig. 2(h) is not a conserved current and therefore it does give the nonvanishing contribution to the $g g \rightarrow W W H$ amplitude. 
[1] G. Aad et al., Study of the spin and parity of the Higgs boson in diboson decays with the ATLAS detector, Eur. Phys. J. C 75, 476 (2015); Erratum, Eur. Phys. J. C 76, 152 (2016).

[2] G. Aad et al., Measurements of the Higgs boson production and decay rates and constraints on its couplings from a combined ATLAS and CMS analysis of the LHC pp collision data at $\sqrt{s}=7$ and $8 \mathrm{TeV}$, J. High Energy Phys. 08 (2016) 045.

[3] A. M. Sirunyan et al., Combined measurements of Higgs boson couplings in proton-proton collisions at $\sqrt{s}=13 \mathrm{TeV}$, Eur. Phys. J. C 79, 421 (2019).

[4] The ATLAS Collaboration, Combined measurements of Higgs boson production and decay using up to $80 \mathrm{fb}^{-1}$ of proton-proton collision data at $\sqrt{s}=13 \mathrm{TeV}$ collected with the ATLAS experiment, 2019, http://cds.cern.ch/record/ 2668375.

[5] I. Anderson et al., Constraining anomalous HVV interactions at proton and lepton colliders, Phys. Rev. D 89, 035007 (2014).

[6] CMS Collaboration, Constraints on anomalous Higgs boson couplings to vector bosons and fermions in production and decay in the $H \rightarrow 4 \ell$ channel, http://cds.cern.ch/record/ 2725543.

[7] M. Aaboud et al., Evidence for the associated production of the Higgs boson and a top quark pair with the ATLAS detector, Phys. Rev. D 97, 072003 (2018).

[8] A. M. Sirunyan et al., Observation of $\mathrm{t} \bar{t} \mathrm{H}$ Production, Phys. Rev. Lett. 120, 231801 (2018).

[9] ATLAS Collaboration, Prospects for measuring Higgs pair production in the channel $H(\rightarrow \gamma \gamma) H(\rightarrow b \bar{b})$ using the ATLAS detector at the HL-LHC, http://cds.cern.ch/ record/1956733.

[10] C.-Y. Chen, Q.-S. Yan, X. Zhao, Y.-M. Zhong, and Z. Zhao, Probing triple-Higgs productions via $4 \mathrm{~b} 2 \gamma$ decay channel at a $100 \mathrm{TeV}$ hadron collider, Phys. Rev. D 93, 013007 (2016).

[11] B. Fuks, J. H. Kim, and S. J. Lee, Scrutinizing the Higgs quartic coupling at a future $100 \mathrm{TeV}$ protonproton collider with taus and b-jets, Phys. Lett. B 771, 354 (2017).

[12] E. Rossi, Measurement of Higgs-boson self-coupling with single-Higgs and double-Higgs production channels, arXiv:2010.05252.

[13] M. McCullough, An indirect model-dependent probe of the Higgs self-coupling, Phys. Rev. D 90, 015001 (2014); Erratum, Phys. Rev. D 92, 039903 (2015).

[14] S. Borowka, C. Duhr, F. Maltoni, D. Pagani, A. Shivaji, and X. Zhao, Probing the scalar potential via double Higgs boson production at hadron colliders, J. High Energy Phys. 04 (2019) 016.

[15] F. Bishara, R. Contino, and J. Rojo, Higgs pair production in vector-boson fusion at the LHC and beyond, Eur. Phys. J. C 77, 481 (2017).

[16] G. Aad et al., Search for the $H H \rightarrow b \bar{b} b \bar{b}$ process via vector-boson fusion production using proton-proton collisions at $\sqrt{s}=13 \mathrm{TeV}$ with the ATLAS detector, J. High Energy Phys. 07 (2020) 108.

[17] D. de Florian and Z. Kunszt, Two photons plus jet at LHC: The NNLO contribution from the $\mathrm{g} g$ initiated process, Phys. Lett. B 460, 184 (1999).
[18] T. Melia, K. Melnikov, R. Rontsch, M. Schulze, and G. Zanderighi, Gluon fusion contribution to $\mathrm{W}+\mathrm{W}-+$ jet production, J. High Energy Phys. 08 (2012) 115.

[19] P. Agrawal and A. Shivaji, Di-vector boson + jet production via gluon fusion at hadron colliders, Phys. Rev. D 86, 073013 (2012).

[20] F. Campanario, Towards pp -> VVjj at NLO QCD: Bosonic contributions to triple vector boson production plus jet, J. High Energy Phys. 10 (2011) 070.

[21] P. Agrawal and A. Shivaji, Production of $\gamma Z g$ and associated processes via gluon fusion at hadron colliders, J. High Energy Phys. 01 (2013) 071.

[22] F. Campanario, Q. Li, M. Rauch, and M. Spira, ZZ+jet production via gluon fusion at the LHC, J. High Energy Phys. 06 (2013) 069.

[23] A. K. Shivaji, Gluon fusion processes at one-loop within the standard model and beyond, Ph.D. thesis, Bhubaneswar, Inst. Phys., 2013.

[24] J. M. Campbell, R. Keith Ellis, E. Furlan, and R. Rntsch, Interference effects for Higgs boson mediated $Z$-pair plus jet production, Phys. Rev. D 90, 093008 (2014).

[25] P. Agrawal and A. Shivaji, Gluon fusion contribution to $V H j$ production at hadron colliders, Phys. Lett. B 741, 111 (2015).

[26] M. Song, W.-G. Ma, R.-Y. Zhang, L. Guo, S.-M. Wang, and L. Han, QCD corrections to associated Higgs boson production with a $W$ boson pair at the LHC, Phys. Rev. D 79, 054016 (2009).

[27] B. Hespel, F. Maltoni, and E. Vryonidou, Higgs and Z boson associated production via gluon fusion in the SM and the 2HDM, J. High Energy Phys. 06 (2015) 065.

[28] E. Gabrielli, B. Mele, F. Piccinini, and R. Pittau, Asking for an extra photon in Higgs production at the LHC and beyond, J. High Energy Phys. 07 (2016) 003.

[29] F. Caola, K. Melnikov, R. Rntsch, and L. Tancredi, QCD corrections to $\mathrm{ZZ}$ production in gluon fusion at the LHC, Phys. Rev. D 92, 094028 (2015).

[30] F. Caola, K. Melnikov, R. Rntsch, and L. Tancredi, QCD corrections to $W^{+} W^{-}$production through gluon fusion, Phys. Lett. B 754, 275 (2016).

[31] J. M. Campbell, R. Keith Ellis, Y. Li, and C. Williams, Predictions for diphoton production at the LHC through NNLO in QCD, J. High Energy Phys. 07 (2016) 148.

[32] F. Caola, M. Dowling, K. Melnikov, R. Rntsch, and L. Tancredi, QCD corrections to vector boson pair production in gluon fusion including interference effects with off-shell Higgs at the LHC, J. High Energy Phys. 07 (2016) 087.

[33] F. Granata, J. M. Lindert, C. Oleari, and S. Pozzorini, NLO $\mathrm{QCD}+\mathrm{EW}$ predictions for $\mathrm{HV}$ and $\mathrm{HV}+$ jet production including parton-shower effects, J. High Energy Phys. 09 (2017) 012.

[34] A. Shivaji, P. Agrawal, and D. Saha, Gluon fusion contribution to $H B B(B=H, \gamma, Z)$ at the LHC, EPJ Web Conf. 129, 00005 (2016).

[35] T. Plehn and M. Rauch, Quartic Higgs coupling at hadron colliders, Phys. Rev. D 72, 053008 (2005).

[36] T. Binoth, S. Karg, N. Kauer, and R. Ruckl, Multi-Higgs boson production in the Standard Model and beyond, Phys. Rev. D 74, 113008 (2006). 
[37] F. Maltoni, E. Vryonidou, and M. Zaro, Top-quark mass effects in double and triple Higgs production in gluon-gluon fusion at NLO, J. High Energy Phys. 11 (2014) 079.

[38] A. Papaefstathiou and K. Sakurai, Triple Higgs boson production at a $100 \mathrm{TeV}$ proton-proton collider, J. High Energy Phys. 02 (2016) 006.

[39] W. Kilian, S. Sun, Q.-S. Yan, X. Zhao, and Z. Zhao, New physics in multi-Higgs boson final states, J. High Energy Phys. 06 (2017) 145.

[40] V. Hirschi and O. Mattelaer, Automated event generation for loop-induced processes, J. High Energy Phys. 10 (2015) 146.

[41] P. Agrawal, D. Saha, and A. Shivaji, Production of $H H H$ and $H H V(V=\gamma, Z)$ at the hadron colliders, Phys. Rev. D 97, 036006 (2018).

[42] M. Mangano and M. Mangano, Physics at the FCC-hh, a $100 \mathrm{TeV}$ pp Collider, Volume 3 of CERN Yellow Reports: Monographs (CERN, Geneva, 2017).

[43] M. Ahmad et al., CEPC-SPPC Preliminary Conceptual Design Report. 1. Physics and Detector 2015, http://cepc .ihep.ac.cn/preCDR/main_preCDR.pdf.

[44] J. Baglio, Next-to-leading order QCD corrections to associated production of a SM Higgs boson with a pair of weak bosons in the POWHEG-BOX, Phys. Rev. D 93, 054010 (2016).

[45] J. Baglio, Gluon fusion and $b \bar{b}$ corrections to $H W^{+} W^{-} / H Z Z$ production in the POWHEG-BOX, Phys. Lett. B 764, 54 (2017).

[46] S. Chatrchyan et al., Measurement of the Polarization of $W$ Bosons with Large Transverse Momenta in $W+$ jets Events at the LHC, Phys. Rev. Lett. 107, 021802 (2011).

[47] G. Aad et al., Measurement of the W boson polarization in top quark decays with the ATLAS detector, J. High Energy Phys. 06 (2012) 088.

[48] M. Aaboud et al., Measurement of $W^{ \pm} Z$ production cross sections and gauge boson polarisation in $p p$ collisions at $\sqrt{s}=13 \mathrm{TeV}$ with the ATLAS detector, Eur. Phys. J. C 79, 535 (2019).

[49] D. Binosi, J. Collins, C. Kaufhold, and L. Theussl, JaxoDraw: A Graphical user interface for drawing Feynman diagrams. Version 2.0 release notes, Comput. Phys. Commun. 180, 1709 (2009).

[50] K. Nishijima, Generalized Furry's theorem for closed loops, Prog. Theor. Phys. 6, 614 (1951).

[51] M. Tanabashi et al., Review of particle physics, Phys. Rev. D 98, 030001 (2018).

[52] G. Aad et al. (ATLAS Collaboration), Combination of searches for Higgs boson pairs in $p p$ collisions at $\sqrt{s}=$ $13 \mathrm{TeV}$ with the ATLAS detector, arXiv:1906.02025.

[53] A. David, A. Denner, M. Duehrssen, M. Grazzini, C. Grojean, G. Passarino, M. Schumacher, M. Spira, G. Weiglein, and M. Zanetti (LHC Higgs Cross Section Working Group), LHC HXSWG interim recommendations to explore the coupling structure of a Higgs-like particle, arXiv:1209.0040.

[54] M. Ghezzi, R. Gomez-Ambrosio, G. Passarino, and S. Uccirati, NLO Higgs effective field theory and $\kappa$-framework, J. High Energy Phys. 07 (2015) 175.

[55] P. Agrawal and G. Ladinsky, Production of two photons and a jet through gluon fusion, Phys. Rev. D 63, 117504 (2001).
[56] J. A. M. Vermaseren, New features of FORM, arXiv:math$\mathrm{ph} / 0010025$.

[57] G. J. van Oldenborgh and J. A. M. Vermaseren, New algorithms for one loop integrals, Z. Phys. C 46, 425 (1990).

[58] A. van Hameren, OneLOop: For the evaluation of one-loop scalar functions, Comput. Phys. Commun. 182, 2427 (2011).

[59] F. Maltoni, K. Paul, T. Stelzer, and S. Willenbrock, Associated production of Higgs and single top at hadron colliders, Phys. Rev. D 64, 094023 (2001).

[60] J. Alwall, R. Frederix, S. Frixione, V. Hirschi, F. Maltoni, O. Mattelaer, H. S. Shao, T. Stelzer, P. Torrielli, and M. Zaro, The automated computation of tree-level and next-toleading order differential cross sections, and their matching to parton shower simulations, J. High Energy Phys. 07 (2014) 079.

[61] S. Veseli, Multidimensional integration in a heterogeneous network environment, Comput. Phys. Commun. 108, 9 (1998).

[62] G. P. Lepage, VEGAS — an adaptive multi-dimensional integration program, Technical Report No. CLNS-447, Cornell Univ. Lab. Nucl. Stud., Ithaca, NY, 1980.

[63] A. Geist, A. Beguelin, J. Dongarra, W. Jiang, R. Manchek, and V. Sunderam, PVM: Parallel Virtual Machine: A Users' Guide and Tutorial for Networked Parallel Computing (MIT Press, Cambridge, MA, USA, 1994).

[64] S. Dulat, T.-J. Hou, J. Gao, M. Guzzi, J. Huston, P. Nadolsky, J. Pumplin, C. Schmidt, D. Stump, and C. P. Yuan, New parton distribution functions from a global analysis of quantum chromodynamics, Phys. Rev. D 93, 033006 (2016).

[65] R. Aaij et al., First Experimental Study of Photon Polarization in Radiative $B_{s}^{0}$ Decays, Phys. Rev. Lett. 118, 021801 (2017); 118, 109901(A) (2017).

[66] F. Legger and T. Schietinger, Photon helicity in $\Lambda_{b} \rightarrow p K \gamma$ decays, Phys. Lett. B 645, 204 (2007); , Erratum, Phys. Lett. B 647, 527 (2007).

[67] G. Hiller, M. Knecht, F. Legger, and T. Schietinger, Photon polarization from helicity suppression in radiative decays of polarized Lambda(b) to spin-3/2 baryons, Phys. Lett. B 649, 152 (2007).

[68] V.D. Orlovsky and V. I. Shevchenko, On the photon polarization in radiative $B \rightarrow \varphi K \gamma$ decay, Phys. Rev. D 77, 093003 (2008).

[69] L. Shchutska, Y. Xie, A. Golutvin, V. Egorychev, V. Shevchenko, and I. Belyaev, Probing the photon polarization in Bs->phi gamma at LHCb, http://cds.cern.ch/record/ 1099116.

[70] L. Oliver, J. C. Raynal, and R. Sinha, Note on new interesting baryon channels to measure the photon polarization in $b \rightarrow s \gamma$, Phys. Rev. D 82, 117502 (2010).

[71] L. M. García Martín, B. Jashal, F. Martínez Vidal, A. Oyanguren, S. Roy, R. Sain, and R. Sinha, Radiative $b$-baryon decays to measure the photon and $b$-baryon polarization, Eur. Phys. J. C 79, 634 (2019).

[72] M. Grossi, J. Novak, B. Kersevan, and D. Rebuzzi, Comparing traditional and deep-learning techniques of kinematic reconstruction for polarization discrimination in vector boson scattering, Eur. Phys. J. C 80, 1144 (2020).

[73] L. D. Landau, On the angular momentum of a system of two photons, Dokl. Akad. Nauk Ser. Fiz. 60, 207 (1948). 
[74] C.-N. Yang, Selection rules for the dematerialization of a particle into two photons, Phys. Rev. 77, 242 (1950).

[75] T. Binoth, M. Ciccolini, N. Kauer, and M. Kramer, Gluoninduced WW background to Higgs boson searches at the LHC, J. High Energy Phys. 03 (2005) 065.
[76] J. M. Campbell, R. Keith Ellis, and C. Williams, Gluon-gluon contributions to $\mathrm{W}+\mathrm{W}-$ production and Higgs interference effects, J. High Energy Phys. 10 (2011) 005 . 